\title{
Regioselective and Enantiospecific Rhodium-Catalyzed Allylic Alkylation Reactions using Copper(I) Enolates: Synthesis of (-)-Sugiresinol Dimethyl Ether
}

\author{
P. Andrew Evans* and David K. Leahy \\ Department of Chemistry, Indiana University, Bloomington, IN 47405
}

Experimental Procedures and Supplemental Data

1-Phenyl-3-(2-phenylethyl)pent-4-en-1-one rac-2a. Racemic GC analysis (HP-1 Methyl Siloxane capillary column) $2^{\circ}: 1^{\circ} \geq 99: 1$; IR (neat) 3063 (m), 3027 (m), 2925 (s), 2857 (m), 1683 (s), 1640 (w), 1598 (s), 1496 (m), 1449 (s) cm ${ }^{-1} ;{ }^{1} \mathrm{H}$ NMR (400 MHz, CDCl $) \delta 7.91$ (d, $J=7.3$ $\mathrm{Hz}, 2 \mathrm{H}), 7.54$ (t, $J=7.4 \mathrm{~Hz}, 1 \mathrm{H}), 7.44$ (t, $J=7.9 \mathrm{~Hz}, 2 \mathrm{H}), 7.28-7.15(\mathrm{~m}, 5 \mathrm{H}), 5.78-5.70(\mathrm{~m}, 1 \mathrm{H})$, $5.06(\mathrm{~d}, J=15.2 \mathrm{~Hz}, 1 \mathrm{H}), 5.05(\mathrm{~d}, J=11.7 \mathrm{~Hz}, 1 \mathrm{H}), 3.03\left(\mathrm{dd}, \mathrm{A}\right.$ of ABX, $J_{A B}=16.3 \mathrm{~Hz}, J_{A X}=$ $7.6 \mathrm{~Hz}, 1 \mathrm{H}$ ), 2.99 (dd, B of ABX, $J_{A B}=16.3 \mathrm{~Hz}, J_{B X}=6.2 \mathrm{~Hz}, 1 \mathrm{H}$ ), 2.82 (sextet, $J=7.1 \mathrm{~Hz}, 1 \mathrm{H}$ ), 2.70 (ddd, A of ABXY, $\left.J_{A B}=13.8 \mathrm{~Hz}, J_{A X}=10.6 \mathrm{~Hz}, J_{A Y}=5.3 \mathrm{~Hz}, 1 \mathrm{H}\right), 2.58$ (ddd, B of ABXY, $\left.J_{A B}=13.8 \mathrm{~Hz}, J_{B X}=10.4 \mathrm{~Hz}, J_{B Y}=6.3 \mathrm{~Hz}, 1 \mathrm{H}\right), 1.87-1.78(\mathrm{~m}, 1 \mathrm{H}), 1.72-1.62(\mathrm{~m}, 1 \mathrm{H}) ;{ }^{13} \mathrm{C} \mathrm{NMR}$ (100 MHz, $\left.\mathrm{CDCl}_{3}\right) \delta 199.40$ (e), 142.43 (e), 141.31 (o), 137.49 (e), 133.16 (o), 128.77 (o), 128.58 (o), 128.54 (o), 128.30 (o), 125.95 (o), 115.70 (e), 44.12 (e), 39.74 (o), 36.61 (e), 33.66 (e); HRMS (CI, $\mathrm{M}^{+}$) calcd for $\mathrm{C}_{19} \mathrm{H}_{20} \mathrm{O} 264.1514$, found 264.1510.

(S)-1-Phenyl-3-(2-phenylethyl)pent-4-en-1-one $(\boldsymbol{S})$-2a. $[\alpha]_{\mathrm{D}}{ }^{26}-10.9\left(\mathrm{c}=1.08, \mathrm{CHCl}_{3}\right)$; Chiral HPLC analysis (Diacel ${ }^{\circledR}$ AD column) $e e \geq 99 \%$; Racemic GC analysis (HP-1 Methyl Siloxane capillary column) $2^{\circ}: 1^{\circ} \geq 99: 1$; IR (neat) $3063(\mathrm{~m}), 3027$ (m), 2925 (s), 2857 (m), 1683 (s), 1640 (w), 1598 (s), 1496 (m), 1449 (s) cm ${ }^{-1}$; ${ }^{1} \mathrm{H}$ NMR (400 MHz, $\mathrm{CDCl}_{3}$ ) $\delta 7.91$ (d, $J=7.3 \mathrm{~Hz}, 2 \mathrm{H}$ ), $7.54(\mathrm{t}, J=7.4 \mathrm{~Hz}, 1 \mathrm{H}), 7.44$ (t, $J=7.9 \mathrm{~Hz}, 2 \mathrm{H}), 7.28-7.15$ (m, 5H), 5.78-5.70 (m, 1H), 5.06 (d, $J$ $=15.2 \mathrm{~Hz}, 1 \mathrm{H}), 5.05(\mathrm{~d}, J=11.7 \mathrm{~Hz}, 1 \mathrm{H}), 3.03\left(\mathrm{dd}, \mathrm{A}\right.$ of ABX, $\left.J_{A B}=16.3 \mathrm{~Hz}, J_{A X}=7.6 \mathrm{~Hz}, 1 \mathrm{H}\right)$, $2.99\left(\mathrm{dd}, \mathrm{B}\right.$ of ABX, $\left.J_{A B}=16.3 \mathrm{~Hz}, J_{B X}=6.2 \mathrm{~Hz}, 1 \mathrm{H}\right), 2.82$ (sextet, $J=7.1 \mathrm{~Hz}, 1 \mathrm{H}$ ), 2.70 (ddd, A of ABXY, $J_{A B}=13.8 \mathrm{~Hz}, J_{A X}=10.6 \mathrm{~Hz}, J_{A Y}=5.3 \mathrm{~Hz}, 1 \mathrm{H}$ ), $2.58\left(\mathrm{ddd}, \mathrm{B}\right.$ of ABXY, $J_{A B}=13.8 \mathrm{~Hz}$, $\left.J_{B X}=10.4 \mathrm{~Hz}, J_{B Y}=6.3 \mathrm{~Hz}, 1 \mathrm{H}\right), 1.87-1.78(\mathrm{~m}, 1 \mathrm{H}), 1.72-1.62(\mathrm{~m}, 1 \mathrm{H})$.

3-Methyl-1-phenylpent-4-en-1-one rac-2b. Racemic GC analysis (HP-1 Methyl Siloxane capillary column) $2^{\circ}: 1^{\circ} \geq 99: 1 ;$ IR (neat) 3082 (w), 2963 (m), 2929 (w), 2857 (w), 1686 (s), 
$1641(\mathrm{w}), 1598(\mathrm{~m}), 1449$ (s) $\mathrm{cm}^{-1} ;{ }^{1} \mathrm{H}$ NMR (400 MHz, $\left.\mathrm{CDCl}_{3}\right) \delta$ 7.94-7.92 (m, 2H), $7.54(\mathrm{t}, J=$ $7.4 \mathrm{~Hz}, 1 \mathrm{H}), 7.44(\mathrm{t}, J=7.6 \mathrm{~Hz}, 2 \mathrm{H}), 5.83(\mathrm{ddd}, J=17.0,10.5,6.5 \mathrm{~Hz}, 1 \mathrm{H}), 5.01$ (dt, $J=17.3$, $1.2 \mathrm{~Hz}, 1 \mathrm{H}), 4.94(\mathrm{~d}, J=10.5 \mathrm{~Hz}, 1 \mathrm{H}), 3.01\left(\mathrm{dd}, \mathrm{A}\right.$ of $\left.\mathrm{ABX}, J_{A B}=18.2 \mathrm{~Hz}, J_{A X}=9.0 \mathrm{~Hz}, 1 \mathrm{H}\right)$, 2.92-2.85 (m, 1H), $2.88\left(\mathrm{dd}, \mathrm{B}\right.$ of ABX, $\left.J_{A B}=18.0 \mathrm{~Hz}, J_{B X}=6.9 \mathrm{~Hz}, 1 \mathrm{H}\right), 1.08(\mathrm{~d}, J=6.4 \mathrm{~Hz}$, 3H); ${ }^{13} \mathrm{C}$ NMR (100 MHz, $\mathrm{CDCl}_{3}$ ) $\delta 199.59$ (e), 143.27 (o), 137.51 (e), 133.18 (o), 128.79 (o), 128.32 (o), 113.26 (e), 45.36 (e), 33.82 (o), 20.02 (o); HRMS (CI, M ) calcd for $\mathrm{C}_{12} \mathrm{H}_{14} \mathrm{O}$ 174.1045, found 174.1048 .

1-Phenyl-3-propylpent-4-en-1-one rac-2c. Racemic GC analysis (HP-1 Methyl Siloxane capillary column) $2^{\circ}: 1^{\circ} \geq 99: 1 ;$ IR (neat) 3078 (w), 2958 (s), 2930 (s), 2872 (m), 1687 (s), 1640 (w), 1598 (m), 1449 (s) cm ${ }^{-1}$; ${ }^{1} \mathrm{H}$ NMR (400 MHz, $\mathrm{CDCl}_{3}$ ) $\delta$ 7.93-7.91 (m, 2H), 7.53 (tt, J= 7.4, $1.4 \mathrm{~Hz}, 1 \mathrm{H}), 7.44$ (t, $J=7.6 \mathrm{~Hz}, 2 \mathrm{H}), 5.66$ (ddd, $J=17.2,10.2,8.3 \mathrm{~Hz}, 1 \mathrm{H}), 4.99-4.94$ (m, 2H), $2.95(\mathrm{~d}, J=6.8 \mathrm{~Hz}, 2 \mathrm{H}), 2.74$ (sextet, $J=6.9 \mathrm{~Hz}, 1 \mathrm{H}), 1.47-1.22(\mathrm{~m}, 4 \mathrm{H}), 0.87$ (t, $J=6.9 \mathrm{~Hz}$, 3H); ${ }^{13} \mathrm{C}$ NMR (100 MHz, $\mathrm{CDCl}_{3}$ ) $\delta 199.75$ (e), 141.79 (o), 137.59 (e), 133.11 (o), 128.77 (o), 128.33 (o), 114.91 (e), 44.15 (e), 39.76 (o), 37.13 (e), 20.44 (e), 14.26 (o); HRMS (CI, M ) calcd for $\mathrm{C}_{14} \mathrm{H}_{18} \mathrm{O} 202.1358$, found 202.1368.

1-Phenyl-3-vinyloct-7-en-1-one rac-2d. Racemic GC analysis (HP-1 Methyl Siloxane capillary

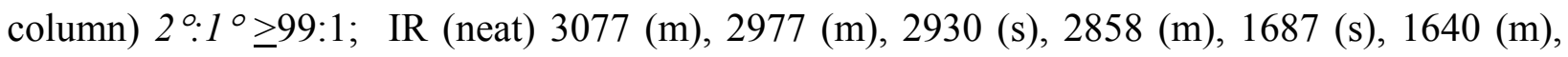
$1597(\mathrm{~m}), 1449(\mathrm{~m}) \mathrm{cm}^{-1} ;{ }^{1} \mathrm{H}$ NMR $\left(400 \mathrm{MHz} \mathrm{CDCl}_{3}\right) \delta$ 7.93-7.90 (m, 2H), 7.53 (tt, J= 7.4, 1.5 $\mathrm{Hz}, 1 \mathrm{H}), 7.43$ (t, $J=7.6 \mathrm{~Hz}, 2 \mathrm{H}), 5.78$ (ddt, $J=17.1,10.2,6.7 \mathrm{~Hz}, 1 \mathrm{H}), 5.66$ (ddd, $J=17.2,10.3$, $8.3 \mathrm{~Hz}, 1 \mathrm{H}), 5.01-4.90(\mathrm{~m}, 4 \mathrm{H}), 2.98\left(\mathrm{dd}, \mathrm{A}\right.$ of ABX, $\left.J_{A B}=16.0 \mathrm{~Hz}, J_{A X}=7.4 \mathrm{~Hz}, 1 \mathrm{H}\right), 2.94(\mathrm{dd}$, B of $\left.\mathrm{ABX}, J_{A B}=16.0 \mathrm{~Hz}, J_{B X}=6.4 \mathrm{~Hz}, 1 \mathrm{H}\right), 2.79-2.69(\mathrm{~m}, 1 \mathrm{H}), 2.09-1.96(\mathrm{~m}, 2 \mathrm{H}), 1.53-1.29$ (m, 4H); ${ }^{13} \mathrm{C}$ NMR (100 MHz, $\mathrm{CDCl}_{3}$ ) $\delta 199.58$ (e), 141.61 (o), 138.95 (o), 137.55 (e), 133.11 (o), 128.76 (o), 128.30 (o), 115.10 (e), 114.67 (e), 44.12 (e), 39.82 (o), 34.31 (e), 33.89 (e), 26.59 (e); HRMS (CI, $\mathrm{M}^{+}$) calcd for $\mathrm{C}_{16} \mathrm{H}_{20} \mathrm{O} 228.1514$, found 228.1508 .

3-Cyclohexyl-1-phenylpent-4-en-1-one rac-2e. Racemic GC analysis (HP-1 Methyl Siloxane capillary column) $2^{\circ}: 1^{\circ} \geq 99: 1 ;$ IR (neat) 3074 (m), 2975 (m), 2924 (s), 2852 (s), 1683 (s), 1639 (m), 1598 (s), 1580 (m), 1448 (m) cm ${ }^{-1}$; ${ }^{1} \mathrm{H}$ NMR (400 MHz, $\mathrm{CDCl}_{3}$ ) $\delta$ 7.92-7.90 (m, 2H), 7.53 (tt, $J=7.4,1.5 \mathrm{~Hz}, 1 \mathrm{H}), 7.43(\mathrm{t}, J=7.6 \mathrm{~Hz}, 2 \mathrm{H}), 5.67(\mathrm{ddd}, J=17.2,10.2,8.8 \mathrm{~Hz}, 1 \mathrm{H}), 4.95$ (dd, $J=10.3,1.7 \mathrm{~Hz}, 1 \mathrm{H}), 4.88(\mathrm{ddd}, J=17.1,1.7,0.8 \mathrm{~Hz}, 1 \mathrm{H}), 3.05\left(\mathrm{dd}, \mathrm{A}\right.$ of $\mathrm{ABX}, J_{A B}=15.7 \mathrm{~Hz}$, 
$\left.J_{A X}=5.2 \mathrm{~Hz}, 1 \mathrm{H}\right), 2.94\left(\mathrm{dd}, \mathrm{B}\right.$ of $\left.\mathrm{ABX}, J_{A B}=15.6 \mathrm{~Hz}, J_{B X}=8.6 \mathrm{~Hz}, 1 \mathrm{H}\right), 2.60(\mathrm{tt}, J=8.6,5.4 \mathrm{~Hz}$, $1 \mathrm{H}), 1.73-1.60(\mathrm{~m}, 5 \mathrm{H}), 1.39-1.30(\mathrm{~m}, 1 \mathrm{H}), 1.27-0.91(\mathrm{~m}, 5 \mathrm{H}) ;{ }^{13} \mathrm{C} \mathrm{NMR}\left(100 \mathrm{MHz}, \mathrm{CDCl}_{3}\right) \delta$ 200.16 (e), 139.90 (o), 137.62 (e), 133.03 (o), 128.74 (o), 128.33 (o), 115.82 (e), 45.73 (o), 41.84 (o), 41.17 (e), 31.14 (e), 29.89 (e), 26.75 (e), 26.71 (e); HRMS (CI, M+H ${ }^{+}$) calcd for $\mathrm{C}_{17} \mathrm{H}_{23} \mathrm{O}$ 243.1749 , found 243.1760.

3-Isopropyl-1-phenylpent-4-en-1-one rac-2f. Racemic GC analysis (HP-1 Methyl Siloxane capillary column) $2^{\circ}: 1^{\circ} \geq 99: 1$; IR (neat) 3077 (w), 2960 (s), 2932 (m), 2874 (m), 1687 (s), 1639

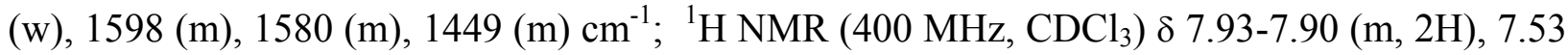
(tt, $J=7.4,1.5 \mathrm{~Hz}, 1 \mathrm{H}), 7.44(\mathrm{t}, J=7.6 \mathrm{~Hz}, 2 \mathrm{H}), 5.67(\mathrm{ddd}, J=17.2,10.3,8.6 \mathrm{~Hz}, 1 \mathrm{H}), 4.98(\mathrm{dd}$, $J=10.3,1.7 \mathrm{~Hz}, 1 \mathrm{H}), 4.92(\mathrm{ddd}, J=17.2,1.7,0.9 \mathrm{~Hz}, 1 \mathrm{H}), 3.01\left(\mathrm{dd}, \mathrm{A}\right.$ of ABX, $J_{A B}=15.6 \mathrm{~Hz}$, $\left.J_{A X}=5.8 \mathrm{~Hz}, 1 \mathrm{H}\right), 2.96\left(\mathrm{dd}, \mathrm{B}\right.$ of $\left.\mathrm{ABX}, J_{A B}=15.7 \mathrm{~Hz}, J_{B X}=8.0 \mathrm{~Hz}, 1 \mathrm{H}\right), 2.61(\mathrm{tt}, J=8.2,5.6 \mathrm{~Hz}$, 1H), 1.72 (double septet, $J=6.7,5.5 \mathrm{~Hz}, 1 \mathrm{H}), 0.92$ (d, $J=6.8 \mathrm{~Hz}, 3 \mathrm{H}), 0.89$ (d, $J=6.7 \mathrm{~Hz}, 3 \mathrm{H})$; ${ }^{13} \mathrm{C}$ NMR (100 MHz, $\mathrm{CDCl}_{3}$ ) $\delta 200.09$ (e), 139.25 (o), 137.65 (e), 133.07 (o), 128.77 (o), 128.34 (o), 116.14 (e), 46.12 (o), 41.24 (e), 31.69 (o), 20.67 (o), 19.07 (o); HRMS (CI, M ${ }^{+}$) calcd for $\mathrm{C}_{14} \mathrm{H}_{18} \mathrm{O} 202.1358$, found 202.1355 .

3-Isobutyl-1-phenylpent-4-en-1-one rac-2g. Racemic GC analysis (HP-1 Methyl Siloxane capillary column) $2^{\circ}: 1^{\circ}=19: 1$; IR (neat) 3077 (w), 2956 (s), 2928 (s), 2869 (m), 1687 (s), 1641

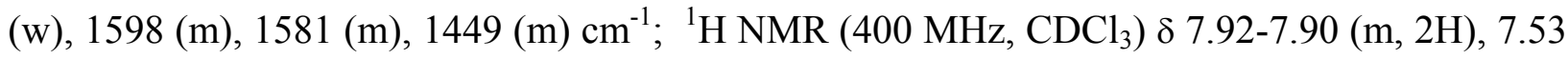
(tt, $J=7.4,1.5 \mathrm{~Hz}, 1 \mathrm{H}), 7.44$ (t, $J=7.6 \mathrm{~Hz}, 2 \mathrm{H}), 5.63(\mathrm{ddd}, J=17.2,10.2,8.4 \mathrm{~Hz}, 1 \mathrm{H}), 4.98$ (d, $J=17.9 \mathrm{~Hz}, 1 \mathrm{H}), 4.94(\mathrm{dd}, J=10.3,1.7 \mathrm{~Hz}, 1 \mathrm{H}), 2.95\left(\mathrm{dd}, \mathrm{A}\right.$ of $\mathrm{ABX}, J_{A B}=15.8 \mathrm{~Hz}, J_{A X}=7.7$ $\mathrm{Hz}, 1 \mathrm{H}), 2.91\left(\mathrm{dd}, \mathrm{B}\right.$ of ABX, $\left.J_{A B}=15.8 \mathrm{~Hz}, J_{B X}=5.9 \mathrm{~Hz}, 1 \mathrm{H}\right), 2.87-2.78(\mathrm{~m}, 1 \mathrm{H}), 1.66-1.52(\mathrm{~m}$, 1H), 1.29 (ddd, A of ABXY, $\left.J_{A B}=13.4 \mathrm{~Hz}, J_{A X}=9.3 \mathrm{~Hz}, J_{A Y}=5.1 \mathrm{~Hz}, 1 \mathrm{H}\right), 1.22$ (ddd, B of $\left.\mathrm{ABXY}, J_{A B}=13.4 \mathrm{~Hz}, J_{B X}=9.2 \mathrm{~Hz}, J_{B Y}=5.1 \mathrm{~Hz}, 1 \mathrm{H}\right), 0.87(\mathrm{~d}, J=6.6 \mathrm{~Hz}, 3 \mathrm{H}), 0.86(\mathrm{~d}, J=6.6$ $\mathrm{Hz}, 3 \mathrm{H}) ;{ }^{13} \mathrm{C} \mathrm{NMR}\left(100 \mathrm{MHz}, \mathrm{CDCl}_{3}\right) \delta 199.70$ (e), 141.84 (o), 137.62 (e), 133.11 (o), 128.77 (o), 128.31 (o), 114.93 (e), 44.62 (e), 44.34 (e), 38.01 (o), 25.60 (o), 23.70 (o), 21.88 (o); HRMS $\left(\mathrm{CI}, \mathrm{M}^{+}\right.$) calcd for $\mathrm{C}_{15} \mathrm{H}_{20} \mathrm{O} 216.1514$, found 216.1515 .

3-(3'-Methylbutyl)-1-phenylpent-4-en-1-one rac-2h. Racemic GC analysis (HP-1 Methyl Siloxane capillary column) $2^{\circ}: 1^{\circ} \geq 99: 1 ;$ IR (neat) 3078 (w), 2955 (s), 2927 (s), 2870 (m), 1688 (s), 1641 (w), 1598 (m), $1581(\mathrm{w}), 1449(\mathrm{~m}) \mathrm{cm}^{-1}$; ${ }^{1} \mathrm{H}$ NMR (400 MHz, $\mathrm{CDCl}_{3}$ ) $\delta$ 7.93-7.91 (m, 
2H), $7.53(\mathrm{tt}, J=7.4,1.5 \mathrm{~Hz}, 1 \mathrm{H}), 7.44$ (t, $J=7.6 \mathrm{~Hz}, 2 \mathrm{H}), 5.66$ (ddd, $J=17.1,10.4,8.3 \mathrm{~Hz}$, 1H), $4.96(\mathrm{dt}, J=17.9,1.3 \mathrm{~Hz}, 1 \mathrm{H}), 4.95(\mathrm{dd}, J=9.7,1.5 \mathrm{~Hz}, 1 \mathrm{H}), 2.96(\mathrm{~d}, J=7.0 \mathrm{~Hz}, 2 \mathrm{H}), 2.69$ $(\mathrm{dq}, J=14.9,7.1 \mathrm{~Hz}, 1 \mathrm{H}), 1.54-1.41(\mathrm{~m}, 2 \mathrm{H}), 1.37-1.28(\mathrm{~m}, 1 \mathrm{H}), 1.24-1.10(\mathrm{~m}, 2 \mathrm{H}), 0.85(\mathrm{~d}, J=$ $6.6 \mathrm{~Hz}, 3 \mathrm{H}), 0.84$ (d, $J=6.6 \mathrm{~Hz}, 3 \mathrm{H}) ;{ }^{13} \mathrm{C} \mathrm{NMR}\left(100 \mathrm{MHz}, \mathrm{CDCl}_{3}\right) \delta 199.75$ (e), 141.88 (o), 137.60 (e), 133.11 (o), 128.77 (o), 128.33 (o), 114.94 (e), 44.18 (e), 40.17 (o), 36.50 (e), 32.72 (e), 28.25 (o), 23.01 (o), 22.64 (o); HRMS (CI, M ) calcd for $\mathrm{C}_{16} \mathrm{H}_{22} \mathrm{O}$ 230.1671, found 230.1672 .

1,3-Diphenylpent-4-en-1-one rac-2i. Racemic GC analysis (HP-1 Methyl Siloxane capillary column) $2^{\circ}: 1^{\circ} \geq 99: 1$; IR (neat) $3083(\mathrm{~m}), 3062(\mathrm{~m}), 3028(\mathrm{~m}), 1683(\mathrm{~s}), 1638(\mathrm{~m}), 1598(\mathrm{~m})$, $1581(\mathrm{~m}), 1449(\mathrm{~s}) \mathrm{cm}^{-1} ;{ }^{1} \mathrm{H}$ NMR $\left(400 \mathrm{MHz}, \mathrm{CDCl}_{3}\right) \delta 7.91-7.89$ (m, 2H), 7.52 (tt, J= 7.4, 1.4 $\mathrm{Hz}, 1 \mathrm{H}), 7.41(\mathrm{t}, J=7.6 \mathrm{~Hz}, 2 \mathrm{H}), 7.29-7.22(\mathrm{~m}, 4 \mathrm{H}), 7.17(\mathrm{tt}, J=7.0,1.8 \mathrm{~Hz}, 1 \mathrm{H}), 6.02$ (ddd, $J=$ $17.2,10.4,6.7 \mathrm{~Hz}, 1 \mathrm{H}), 5.04(\mathrm{dt}, J=10.3,1.2 \mathrm{~Hz}, 1 \mathrm{H}), 5.00(\mathrm{dt}, J=17.2,1.3 \mathrm{~Hz}, 1 \mathrm{H}), 4.11(\mathrm{q}, J$ $=7.0 \mathrm{~Hz}, 1 \mathrm{H}), 3.41\left(\mathrm{dd}, \mathrm{A}\right.$ of $\left.\mathrm{ABX}, J_{A B}=16.7 \mathrm{~Hz}, J_{A X}=7.7 \mathrm{~Hz}, 1 \mathrm{H}\right), 3.33\left(\mathrm{dd}, \mathrm{B}\right.$ of $\mathrm{ABX}, J_{A B}=$ $\left.16.7 \mathrm{~Hz}, J_{B X}=6.6 \mathrm{~Hz}, 1 \mathrm{H}\right) ;{ }^{13} \mathrm{C} \mathrm{NMR}\left(100 \mathrm{MHz}, \mathrm{CDCl}_{3}\right) \delta 198.48$ (e), 143.36 (e), 140.86 (o), 137.32 (e), 133.25 (o), 128.80 (o), 128.27 (o), 127.93 (o), 126.76 (o) 114.94 (e), 44.73 (o), 44.22 (e); HRMS (CI, M ${ }^{+}$) calcd for $\mathrm{C}_{17} \mathrm{H}_{16} \mathrm{O} 236.1201$, found 236.1194 .

3-(2'-Naphthyl)-1-phenylpent-4-en-1-one rac-2j. Racemic GC analysis (HP-1 Methyl Siloxane capillary column) $2^{\circ}: 1^{\circ} \geq 99: 1$; IR (neat) 3056 (m), 2978 (w), 2897 (w), 1686 (s), 1633 (w), $1598(\mathrm{~m}), 1580(\mathrm{w}), 1448(\mathrm{~m}) \mathrm{cm}^{-1} ;{ }^{1} \mathrm{H} \mathrm{NMR}\left(400 \mathrm{MHz}, \mathrm{CDCl}_{3}\right) \delta 7.93$ (dd, J=7.4, 1.3 $\mathrm{Hz}, 2 \mathrm{H}), 7.79-7.76(\mathrm{~m}, 3 \mathrm{H}), 7.69$ (s, 1H), 7.53 (tt, $J=7.4,1.5 \mathrm{~Hz}, 1 \mathrm{H}), 7.45-7.39$ (m, 5H), 6.11 $(\mathrm{ddd}, J=17.1,10.3,6.7 \mathrm{~Hz}, 1 \mathrm{H}), 5.10(\mathrm{~d}, J=10.2 \mathrm{~Hz}, 1 \mathrm{H}), 5.07$ (d, $J=17.2 \mathrm{~Hz}, 1 \mathrm{H}), 4.31$ (q, $J$ $=6.9 \mathrm{~Hz}, 1 \mathrm{H}), 3.53\left(\mathrm{dd}, \mathrm{A}\right.$ of $\left.\mathrm{ABX}, J_{A B}=16.7 \mathrm{~Hz}, J_{A X}=7.5 \mathrm{~Hz}, 1 \mathrm{H}\right), 3.46\left(\mathrm{dd}, \mathrm{B}\right.$ of $\mathrm{ABX}, J_{A B}=$ $\left.16.8 \mathrm{~Hz}, J_{B X}=6.7 \mathrm{~Hz}, 1 \mathrm{H}\right) ;{ }^{13} \mathrm{C} \mathrm{NMR}\left(100 \mathrm{MHz}, \mathrm{CDCl}_{3}\right) \delta 198.42$ (e), 140.81 (o), 137.33 (e), 133.78 (e), 133.30 (o), 132.56 (e), 128.83 (o), 128.50 (o), 128.30 (o) 127.91 (o), 127.81 (o), 126.54 (o), 126.28 (o), 126.24 (o), 125.74 (o) 115.24 (e), 44.77 (o), 44.16 (e); HRMS (CI, M ) calcd for $\mathrm{C}_{21} \mathrm{H}_{18} \mathrm{O} 286.1358$, found 286.1355 .

3-Benzyl-1-phenylpent-4-en-1-one rac-2k. Racemic GC analysis (HP-1 Methyl Siloxane capillary column) $2^{\circ}: 1^{\circ}=74: 1$; IR (neat) $3083(\mathrm{~m}), 3063(\mathrm{~m}), 3027(\mathrm{~m}), 2923(\mathrm{~m}), 1683(\mathrm{~s})$, 1641 (m), 1598 (s), 1581 (s), 1495 (s), 1449 (s) cm ${ }^{-1} ;{ }^{1} \mathrm{H}$ NMR (400 MHz, $\mathrm{CDCl}_{3}$ ) $\delta$ 7.86-7.84 
$(\mathrm{m}, 2 \mathrm{H}), 7.52(\mathrm{tt}, J=7.4,1.4 \mathrm{~Hz}, 1 \mathrm{H}), 7.42(\mathrm{t}, J=7.4 \mathrm{~Hz}, 2 \mathrm{H}), 7.28-7.25(\mathrm{~m}, 2 \mathrm{H}), 7.20-7.17(\mathrm{~m}$, 3H), 5.78 (ddd, $J=16.9,10.6,7.7 \mathrm{~Hz}, 1 \mathrm{H}), 4.94$ (d, $J=9.8 \mathrm{~Hz}, 1 \mathrm{H}), 4.93$ (dt, $J=18.1,1.2 \mathrm{~Hz}$, 1H), 3.09 (sextet, $J=7.2 \mathrm{~Hz}, 1 \mathrm{H}), 2.99$ (dd, A of ABX, $\left.J_{A B}=16.4 \mathrm{~Hz}, J_{A X}=5.9 \mathrm{~Hz}, 1 \mathrm{H}\right), 2.95$ $\left(\mathrm{dd}, \mathrm{B}\right.$ of $\left.\mathrm{ABX}, J_{A B}=16.2 \mathrm{~Hz}, J_{B X}=7.7 \mathrm{~Hz}, 1 \mathrm{H}\right), 2.75(\mathrm{~d}, J=7.1 \mathrm{~Hz}, 2 \mathrm{H}) ;{ }^{13} \mathrm{C} \mathrm{NMR}(100 \mathrm{MHz}$, $\left.\mathrm{CDCl}_{3}\right) \delta 199.49$ (e), 140.99 (o), 139.91 (e), 137.44 (e), 133.18 (o), 129.59 (o), 128.77 (o), 128.48 (o), 128.27 (o) 126.37 (o), 115.15 (e), 42.70 (e), 41.25 (e), 41.10 (o); HRMS (CI, M+H') calcd for $\mathrm{C}_{18} \mathrm{H}_{19} \mathrm{O} 251.1436$, found 251.1428 .

1-Phenyl-3-vinylpent-4-en-1-one rac-21. Racemic GC analysis (HP-1 Methyl Siloxane capillary column) $2^{\circ}: 1^{\circ}=28: 1$; IR (neat) $3081(\mathrm{w}), 2980(\mathrm{w}), 1688(\mathrm{~s}), 1635(\mathrm{w}), 1598(\mathrm{~m})$, $1580(\mathrm{w}), 1449(\mathrm{~m}) \mathrm{cm}^{-1} ;{ }^{1} \mathrm{H}$ NMR $\left(400 \mathrm{MHz} \mathrm{CDCl}_{3}\right) \delta$ 7.94-7.92 (m, 2H), 7.54 (tt, J=7.4, 1.5 $\mathrm{Hz}, 1 \mathrm{H}), 7.44$ (t, $J=7.6 \mathrm{~Hz}, 2 \mathrm{H}), 5.83$ (ddd, $J=17.3,10.3,7.0 \mathrm{~Hz}, 2 \mathrm{H}), 5.06$ (dt, $J=17.2,1.3$ $\mathrm{Hz}, 2 \mathrm{H}), 5.03$ (dt, $J=10.2,1.2 \mathrm{~Hz}, 2 \mathrm{H}), 3.50$ (triple pentet, $J=7.0,1.1, \mathrm{~Hz}, 1 \mathrm{H}), 3.09$ (d, $J=7.0$ $\mathrm{Hz}, 2 \mathrm{H}) ;{ }^{13} \mathrm{C} \mathrm{NMR}\left(100 \mathrm{MHz}, \mathrm{CDCl}_{3}\right) \delta 198.74$ (e), 139.78 (o), 137.40 (e), 133.26 (o), 128.82 (o), 128.32 (o), 115.29 (e), 43.01 (e), 42.99 (o); HRMS (CI, $\mathrm{M}^{+}$) calcd for $\mathrm{C}_{13} \mathrm{H}_{14} \mathrm{O}$ 186.1045, found 186.1047 .

3-(tert-Butyldimethylsilyloxymethyl)-1-phenylpent-4-en-1-one rac-2m. Racemic GC analysis (HP-1 Methyl Siloxane capillary column) 20:1º 50:1; IR (neat) 3081 (w), 2955 (s), 2929 (s), 2896 (m), 2857 (s), 1687 (s), 1642 (w), 1598 (m), 1581 (w), 1471 (m), 1449 (m) cm ${ }^{-1}$; ${ }^{1} \mathrm{H}$ NMR $\left(400 \mathrm{MHz}, \mathrm{CDCl}_{3}\right) \delta$ 7.96-7.94 (m, 2H), $7.53(\mathrm{tt}, J=7.4,1.5 \mathrm{~Hz}, 1 \mathrm{H}), 7.43(\mathrm{t}, J=7.6 \mathrm{~Hz}, 2 \mathrm{H})$, $5.78(\mathrm{ddd}, J=17.6,10.2,7.4 \mathrm{~Hz}, 1 \mathrm{H}), 5.08-5.01(\mathrm{~m}, 2 \mathrm{H}), 3.67\left(\mathrm{dd}, \mathrm{A}\right.$ of ABX, $J_{A B}=9.8 \mathrm{~Hz}, J_{A X}$ $=4.8 \mathrm{~Hz}, 1 \mathrm{H}), 3.55\left(\mathrm{dd}, \mathrm{B}\right.$ of $\left.\mathrm{ABX}, J_{A B}=9.9 \mathrm{~Hz}, J_{B X}=6.7 \mathrm{~Hz}, 1 \mathrm{H}\right), 3.30\left(\mathrm{dd}, \mathrm{A}\right.$ of $\mathrm{ABX}, J_{A B}=$ $\left.15.5, J_{A X}=5.1 \mathrm{~Hz}, 1 \mathrm{H}\right), 2.94($ sextet, $J=6.5 \mathrm{~Hz}, 1 \mathrm{H}), 2.87\left(\mathrm{dd}, \mathrm{B}\right.$ of $\mathrm{ABX}, J_{A B}=15.5, J_{B X}=7.8$ $\mathrm{Hz}, 1 \mathrm{H}), 0.86(\mathrm{~s}, 9 \mathrm{H}), 0.02(\mathrm{~s}, 3 \mathrm{H}), 0.00(\mathrm{~s}, 3 \mathrm{H}) ;{ }^{13} \mathrm{C} \mathrm{NMR}\left(100 \mathrm{MHz}, \mathrm{CDCl}_{3}\right) \delta 199.76(\mathrm{e})$, 138.81 (o), 137.51 (e), 133.11 (o), 128.74 (o), 128.39 (o), 116.06 (e), 65.94 (e), 42.36 (o) 40.04 (e), 26.10 (o), 18.51 (e), -5.23 (o); HRMS (CI, $\mathrm{M}+\mathrm{H}^{+}$) calcd for $\mathrm{C}_{18} \mathrm{H}_{29} \mathrm{SiO}_{2}$ 305.1937, found 305.1923.

3-[2'-(Benzyloxy)ethyl]-1-phenylpent-4-en-1-one rac-2n. Racemic GC analysis (HP-1 Methyl Siloxane capillary column) $2^{\circ}: 1^{\circ}=83: 1$; IR (neat) 3065 (m), 3030 (m), 2929 (s), 2859 (s), 2796 (w), 1687 (s), 1640 (m), 1598 (m), 1580 (m), 1496 (m), 1449 (s) cm ${ }^{-1}$; ${ }^{1} \mathrm{H}$ NMR (400 MHz, 
$\left.\mathrm{CDCl}_{3}\right) \delta 7.92-7.90(\mathrm{~m}, 2 \mathrm{H}), 7.53(\mathrm{tt}, J=7.4,1.2 \mathrm{~Hz}, 1 \mathrm{H}), 7.42(\mathrm{t}, J=7.6 \mathrm{~Hz}, 2 \mathrm{H}), 7.33-7.24(\mathrm{~m}$, 5H), 5.69 (ddd, $J=17.7,10.0,7.6 \mathrm{~Hz}, 1 \mathrm{H}), 4.98$ (d, $J=17.2 \mathrm{~Hz}, 1 \mathrm{H}), 4.97$ (dd, $J=10.2,1.5 \mathrm{~Hz}$, $1 \mathrm{H}), 4.47\left(\mathrm{~d}, \mathrm{~A}\right.$ of $\left.\mathrm{AB}, J_{A B}=12.0 \mathrm{~Hz}, 1 \mathrm{H}\right), 4.43\left(\mathrm{~d}, \mathrm{~B}\right.$ of $\left.\mathrm{AB}, J_{A B}=9.9 \mathrm{~Hz}, 1 \mathrm{H}\right), 3.55-3.46(\mathrm{~m}$, $2 \mathrm{H}), 3.08-2.89(\mathrm{~m}, 3 \mathrm{H}), 1.87-1.78(\mathrm{~m}, 1 \mathrm{H}), 1.72-1.63(\mathrm{~m}, 1 \mathrm{H}) ;{ }^{13} \mathrm{C} \mathrm{NMR}\left(100 \mathrm{MHz}, \mathrm{CDCl}_{3}\right) \delta$ 199.36 (e), 141.11 (o), 138.69 (e), 137.46 (e), 133.13 (o), 128.77 (o), 128.55 (o), 128.34 (o), 127.88 (o) 127.72 (o), 115.41 (e), 73.12 (e), 68.38 (e) 43.99 (e), 37.07 (o), 34.58 (e); HRMS (CI, $\mathrm{M}+\mathrm{H}^{+}$) calcd for $\mathrm{C}_{20} \mathrm{H}_{22} \mathrm{O}_{2} 295.1698$, found 295.1702 .

$\{$ 2-[(Benzyloxy)methyl]-cyclohex-3-en-1-yl\}(phenyl)methanone 5a. Trimethyl phosphite (12 $\mu \mathrm{L}, 0.10 \mathrm{mmol})$ was added directly to a red suspension of Wilkinson's catalyst $(23.1 \mathrm{mg}, 0.025$ $\mathrm{mmol})$ in anhydrous THF $(1.0 \mathrm{~mL})$ then stirred under an atmosphere of argon. The catalyst was allowed to form over $c a$. 15 minutes resulting in a light yellow homogeneous solution. Lithium hexamethyldisilyl azide ( $475 \mu \mathrm{L}, 0.475 \mathrm{mmol}, 1.0 \mathrm{M}$ solution in THF) was added dropwise to a suspension of copper(I) iodide (95.6 mg, $0.50 \mathrm{mmol}$, previously dried in vacuo at $160{ }^{\circ} \mathrm{C}$ ) and ketone 4 (87.5 mg, $0.50 \mathrm{mmol})$ in anhydrous THF $(1.5 \mathrm{~mL})$ under an atmosphere of argon and the resulting anion was allowed to form over ca. 2 minutes until a light yellow homogeneous solution was obtained. The catalyst and the enolate solutions were then cooled with stirring to 0 ${ }^{\circ} \mathrm{C}$, and the former were then added via Teflon cannula to the copper enolate solution. The allylic carbonate $10(59.9 \mathrm{mg}, 0.25 \mathrm{mmol})$ was then added via a tared $500 \mu \mathrm{L}$ gastight syringe to the catalyst/enolate mixture, and the reaction was allowed to slowly warm to room temperature over $c a$. 4 hours (t.l.c. control) resulting in a tan heterogeneous solution. The reaction mixture was then quenched with $\mathrm{NH}_{4} \mathrm{Cl}$ solution $(1 \mathrm{ml})$ and partitioned between diethyl ether and saturated aqueous $\mathrm{NH}_{4} \mathrm{Cl}$ solution. The organic layers were combined, dried $\left(\mathrm{MgSO}_{4}\right)$, filtered and concentrated in vacuo to afford a crude oil. Purification by flash chromatography (eluting with 2-3\% ethyl acetate/hexanes) followed by removal of excess 4 in vacuo at $120{ }^{\circ} \mathrm{C}$ furnished the diastereomeric ketones $(64.3 \mathrm{mg}, 76 \%)$ as a colorless oil $(d s=10: 1)$.

The ketones (43.6 mg, $0.13 \mathrm{mmol})$ were then dissolved in $\mathrm{CH}_{2} \mathrm{Cl}_{2}(1.3 \mathrm{ml})$ and stirred under an atmosphere of argon. Grubbs' catalyst $(5.3 \mathrm{mg}, 0.007 \mathrm{mmol})$ was added in a single portion, and the resulting reaction mixture was stirred at room temperature for $c a 1$ hour (t.l.c. control). The reaction mixture was absorbed on to a silica gel column and purified by flash chromatography (eluting with 3-5\% ethyl acetate/hexanes) to afford 5a/b (39.5 mg, 99\%) as a colorless oil $(d s=10: 1)$ : Racemic GC analysis (HP-1 Methyl Siloxane capillary column) $2^{\circ}: 1^{\circ}$ 
$=30: 1, d s=10: 1 ;$ IR (neat) $3062(\mathrm{~m}), 3026(\mathrm{~s}), 2927(\mathrm{~s}), 2859(\mathrm{~s}), 2789(\mathrm{w}), 1682(\mathrm{~s}), 1597(\mathrm{~s})$, $1580(\mathrm{~m}), 1496(\mathrm{~m}), 1448(\mathrm{~s}) \mathrm{cm}^{-1}$; ${ }^{1} \mathrm{H}$ NMR $\left(400 \mathrm{MHz}, \mathrm{CDCl}_{3}\right) \delta 7.95$ (dd, $\left.J=7.3,1.3 \mathrm{~Hz}, 2 \mathrm{H}\right)$, 7.54 (t, $J=7.4 \mathrm{~Hz}, 1 \mathrm{H}), 7.43$ (t, $J=7.7 \mathrm{~Hz}, 2 \mathrm{H}), 7.21-7.18(\mathrm{~m}, 3 \mathrm{H}), 7.13-7.11(\mathrm{~m}, 2 \mathrm{H}), 5.82$ (ddd, $J=9.8,6.6,3.0 \mathrm{~Hz}, 1 \mathrm{H}$ ), 5.67 (dd, $J=10.1,2.0 \mathrm{~Hz}, 1 \mathrm{H}$ ), 4.37 (s, 3H), 3.54 (ddd, $J=11.5$, $8.8,2.8 \mathrm{~Hz}, 1 \mathrm{H}), 3.38\left(\mathrm{dd}, \mathrm{A}\right.$ of ABX, $\left.J_{A B}=9.4 \mathrm{~Hz}, J_{A X}=5.0 \mathrm{~Hz}, 1 \mathrm{H}\right), 3.32\left(\mathrm{dd}, \mathrm{B}\right.$ of ABX, $J_{A B}=$ $\left.9.4 \mathrm{~Hz}, J_{B X}=6.4 \mathrm{~Hz}, 1 \mathrm{H}\right), 3.08-3.05(\mathrm{~m}, 1 \mathrm{H}), 2.18-2.14(\mathrm{~m}, 2 \mathrm{H}), 1.96-1.90(\mathrm{~m}, 1 \mathrm{H}), 1.74-1.66$ (m, 1H); ${ }^{13} \mathrm{C}$ NMR (100 MHz, $\left.\mathrm{CDCl}_{3}\right) \delta 203.60$ (e), 138.39 (e), 137.10 (e), 132.99 (o), 128.75 (o), 128.51 (o), 128.37 (o), 128.23 (o), 127.73 (o) 127.63 (o), 127.56 (o), 73.07 (e), 73.02 (e) 44.03 (o), 38.32 (o), 26.48 (e), 25.00 (e); HRMS (CI, $\mathrm{M}+\mathrm{H}^{+}$) calcd for $\mathrm{C}_{21} \mathrm{H}_{22} \mathrm{O}_{2}$ 306.1620, found 306.1617 .

(R)-1-(4'-Methoxyphenyl)prop-2-en-1-ol ( $\boldsymbol{R})$-6. Titanium(IV) isopropoxide (2.2 $\mathrm{ml}, 7.5 \mathrm{mmol})$ was added to a $-23{ }^{\circ} \mathrm{C}$ solution of (-)-dicyclohexyl-D-tartrate $(3.54 \mathrm{~g}, 11.25 \mathrm{mmol})$ and crushed $4 \AA$ molecular sieves $\left(3.65 \mathrm{~g}, 30\right.$ weight $\%$, dried in vacuo at $160{ }^{\circ} \mathrm{C}$ for $c a .12$ hours prior to addition) in $\mathrm{CH}_{2} \mathrm{Cl}_{2}$ (300 ml, $0.25 \mathrm{M}$ in substrate), and allowed to stir under an atmosphere of nitrogen at $-23{ }^{\circ} \mathrm{C}$ for 30 minutes. The reaction was then treated with tert-butyl hydroperoxide (16.5 ml, $82.5 \mathrm{mmol}, 5-6 \mathrm{M}$ solution in decane, dried with $4 \AA$ molecular sieves for $c a .3$ hours prior to addition) followed by rac-6 (12.29 $\mathrm{g}, 75 \mathrm{mmol})$, and stirred under an atmosphere of nitrogen at $-23{ }^{\circ} \mathrm{C}$ (maintained by a constant temperature bath using a NesLab Cryocool) until the enantiomeric excess was $\geq 95 \%$ ( 9 days, monitored by HLPC). The reaction was then warmed to $0{ }^{\circ} \mathrm{C}$ and poured into a freshly prepared aqueous solution of ferrous sulfate heptahydrate $(33 \mathrm{~g}, 120 \mathrm{mmol})$, and tartaric acid $(10 \mathrm{~g}, 60 \mathrm{mmol})$ in deionized water $(100 \mathrm{ml})$ at $0{ }^{\circ} \mathrm{C}$. The two-phase mixture was stirred for 10 minutes, filtered through celite, the phases separated and the aqueous phase extracted with diethyl ether. The combined organic layers were treated with a pre-cooled $\left(0^{\circ} \mathrm{C}\right)$ solution of $30 \%$ aqueous solution of sodium hydroxide $(\mathrm{w} / \mathrm{v})$ in saturated brine. The two-phase mixture was stirred vigorously for 1 hour at $0{ }^{\circ} \mathrm{C}$ then filtered through a pad of celite, before being transferred to a separatory funnel and diluted with water. The phases were separated and the aqueous layer was extracted with diethyl ether. The organic layers were then combined, dried $\left(\mathrm{MgSO}_{4}\right)$, filtered, and concentrated in vacuo to afford a crude oil. Purification by flash chromatography (eluting with $15-20 \%$ ethyl acetate/hexanes) furnished the enantiomerically enriched allylic alcohol $(\boldsymbol{R})-6(4.42 \mathrm{~g}, 36 \%)$ as a colorless oil: $[\alpha]_{\mathrm{D}}{ }^{26}+4.2$ $\left(\mathrm{c}=1.11, \mathrm{CHCl}_{3}\right)$; Chiral HPLC analysis $\left(\right.$ Diacel ${ }^{\circledR} \mathrm{OD}$ column) $e e=95 \%$; IR (neat) 3401 (bs), 
3005 (m), 2957 (m), 2936 (m), 2908 (w), 2837 (m) 1611 (s), 1586 (m), 1512 (s), 1465 (m), 1442 (m), $1420(\mathrm{~m}) \mathrm{cm}^{-1} ;{ }^{1} \mathrm{H}$ NMR $\left(400 \mathrm{MHz}, \mathrm{CDCl}_{3}\right) \delta 7.28(\mathrm{dt}, J=8.6,2.3 \mathrm{~Hz}, 2 \mathrm{H}), 6.87(\mathrm{dt}, J=$ 8.7, $2.5 \mathrm{~Hz}, 2 \mathrm{H}), 6.03$ (ddd, $J=17.1,10.3,5.9 \mathrm{~Hz}, 1 \mathrm{H}), 5.32$ (dt, $J=17.2,1.4 \mathrm{~Hz}, 1 \mathrm{H}), 5.17$ (dt, $J=10.3,1.3 \mathrm{~Hz}, 1 \mathrm{H}), 5.16-5.13(\mathrm{~m}, 1 \mathrm{H}), 3.79(\mathrm{~s}, 3 \mathrm{H}), 1.90(\mathrm{~s}, 1 \mathrm{H}) ;{ }^{13} \mathrm{C} \mathrm{NMR}(100 \mathrm{MHz}$, $\left.\mathrm{CDCl}_{3}\right) \delta 159.40$ (e), 140.54 (o), 135.01 (e), 127.88 (o), 114.99 (e), 114.13 (o), 75.09 (o), 55.51 (o); HRMS (CI, $\mathrm{M}+\mathrm{H}^{+}$) calcd for $\mathrm{C}_{10} \mathrm{H}_{12} \mathrm{O}_{2}$ 164.0837, found 164.0834.

$(\boldsymbol{R})-1,3-B i s(4 '-m e t h o x y p h e n y l) p e n t-4-e n-1-o n e(\boldsymbol{R})-7$. The allylic alcohol $(\boldsymbol{R})-6(81.6 \mathrm{mg}, 0.50$ mmol) was dissolved in anhydrous THF $(2.0 \mathrm{ml})$ and cooled with stirring to $0{ }^{\circ} \mathrm{C}$ under an atmosphere of argon. ${ }^{n}$ Butyllithium $(200 \mu \mathrm{L}, 0.50 \mathrm{mmol}, 2.5 \mathrm{M}$ solution in hexanes) was added dropwise and the reaction stirred for $c a .5$ minutes at $0^{\circ} \mathrm{C}$, before methyl chloroformate $(39 \mu \mathrm{L}$, $0.50 \mathrm{mmol}$ ) was added and the resulting reaction mixture allowed to stir for $c a$. 15 minutes. Trimethyl phosphite $(24 \mu \mathrm{L}, 0.20 \mathrm{mmol})$ was then added directly to a red suspension of Wilkinson's catalyst $(46.1 \mathrm{mg}, 0.05 \mathrm{mmol})$ in anhydrous THF $(1.0 \mathrm{~mL})$ then stirred under an atmosphere of argon. The catalyst was allowed to form over $c a$. 15 minutes resulting in a light yellow homogeneous solution. Lithium hexamethyldisilyl azide $(950 \mu \mathrm{L}, 0.95 \mathrm{mmol}, 1.0 \mathrm{M}$ solution in THF) was then added dropwise to a suspension of copper(I) iodide (190.6 mg, 1.00 mmol, previously dried in vacuo at $160{ }^{\circ} \mathrm{C}$ ) and 4-methoxyacetophenone (150.6 mg, $\left.1.00 \mathrm{mmol}\right)$ in anhydrous THF $(2.0 \mathrm{~mL})$ under an atmosphere of argon and the resulting anion was allowed to form over $c a$. 2 minutes until a light yellow homogeneous solution was obtained. The allylic carbonate, catalyst and enolate solutions were then cooled with stirring to $0{ }^{\circ} \mathrm{C}$, and to the allylic carbonate was added the copper enolate followed by the catalyst solution via Teflon cannula. The reaction mixture was then allowed to slowly warm to room temperature over $c a$. 4 hours (t.l.c. control) resulting in a tan heterogeneous solution. The reaction mixture was then quenched with $\mathrm{NH}_{4} \mathrm{Cl}$ solution $(1 \mathrm{ml})$ and partitioned between diethyl ether and saturated aqueous $\mathrm{NH}_{4} \mathrm{Cl}$ solution. The organic layers were combined, dried $\left(\mathrm{MgSO}_{4}\right)$, filtered and concentrated in vacuo to afford a crude oil. Purification by flash chromatography (eluting with 10\% ethyl acetate/hexanes) followed by removal of excess 4-methoxyacetephenone in vacuo at $160{ }^{\circ} \mathrm{C}$ furnished the $\gamma, \delta$-unsaturated ketone $(\boldsymbol{R})-7(118.0 \mathrm{mg}, 80 \%)$ as a colorless oil: $[\alpha]_{\mathrm{D}}{ }^{25}-8.0(\mathrm{c}=$ 1.09, $\mathrm{CHCl}_{3}$ ); Chiral HPLC analysis (Diacel ${ }^{\circledR} \mathrm{AD}-\mathrm{H}$ column) $e e=95 \%$; Racemic GC analysis

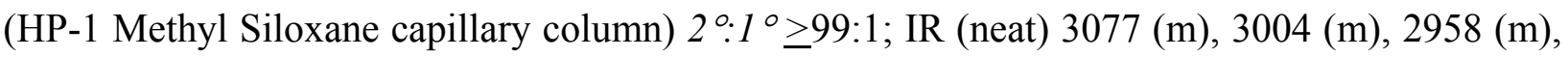
2936 (m), 2907 (m), 2838 (m), 1675 (s), 1636 (m), 1599 (s), 1576 (s), 1509 (s), 1464 (s), 1442 
(m), $1420(\mathrm{~s}) \mathrm{cm}^{-1} ;{ }^{1} \mathrm{H}$ NMR $\left(400 \mathrm{MHz}, \mathrm{CDCl}_{3}\right) \delta 7.90$ (d, $\left.J=8.7 \mathrm{~Hz}, 2 \mathrm{H}\right), 7.15(\mathrm{~d}, J=8.6 \mathrm{~Hz}$, 2H), 6.89 (d, $J=8.9 \mathrm{~Hz}, 2 \mathrm{H}), 6.82$ (d, $J=8.6 \mathrm{~Hz}, 2 \mathrm{H}), 6.01$ (ddd, $J=17.1,10.4,6.7 \mathrm{~Hz}, 1 \mathrm{H})$, $5.02(\mathrm{~d}, J=10.6 \mathrm{~Hz}, 1 \mathrm{H}), 4.98(\mathrm{~d}, J=17.5 \mathrm{~Hz}, 1 \mathrm{H}), 4.05(\mathrm{q}, J=6.9 \mathrm{~Hz}, 1 \mathrm{H}), 3.84(\mathrm{~s}, 3 \mathrm{H}), 3.76$ $(\mathrm{s}, 3 \mathrm{H}), 3.33\left(\mathrm{dd}, \mathrm{A}\right.$ of ABX, $\left.J_{A B}=16.2 \mathrm{~Hz}, J_{A X}=7.5 \mathrm{~Hz}, 1 \mathrm{H}\right), 3.25\left(\mathrm{dd}, \mathrm{B}\right.$ of $\mathrm{ABX}, J_{A B}=16.2$ $\left.\mathrm{Hz}, J_{B X}=6.7 \mathrm{~Hz}, 1 \mathrm{H}\right) ;{ }^{13} \mathrm{C} \mathrm{NMR}\left(100 \mathrm{MHz}, \mathrm{CDCl}_{3}\right) \delta 197.18$ (e), 163.63 (e), $158.36(\mathrm{e}), 141.33$ (o), 135.53 (e), 130.58 (o), 130.46 (e), 128.87 (o), 114.47 (e) 114.15 (o), 113.91 (o), 55.67 (o), 55.44 (o) 44.08 (o), 43.99 (e); $\operatorname{HRMS}\left(\mathrm{CI}, \mathrm{M}+\mathrm{H}^{+}\right.$) calcd for $\mathrm{C}_{19} \mathrm{H}_{20} \mathrm{O}_{3}$ 296.1412, found 296.1411 .

(S,S)-2,4-bis(4'-Methoxyphenyl)-4-oxo-1-(triethylsilyloxymethyl)butyl acetate 8a. To a vigorously stirred solution of methanesulfonamide $(7.1 \mathrm{mg}, 0.075 \mathrm{mmol})$ and AD-mix- $\alpha$ (194.5 $\mathrm{mg}, 3.35 \mathrm{~g} / \mathrm{mmol})$, in tert-butanol $(335 \mu \mathrm{L})$ and deionized water $(335 \mu \mathrm{L})$ under an atmosphere of nitrogen at $0{ }^{\circ} \mathrm{C}$ was added alkene $(\boldsymbol{R})-7(17.1 \mathrm{mg}, 0.058 \mathrm{mmol})$ via tared syringe. The reaction was allowed to warm to room temperature slowly and stirred vigorously for $c a$. 12 hours (t.l.c. control). Sodium sulfite $(232.6 \mathrm{mg}, 1.85 \mathrm{mmol})$ was added at $0{ }^{\circ} \mathrm{C}$ and the reaction stirred for $c a$. 1 hour. The reaction mixture was partitioned between ethyl acetate and $1 \mathrm{~N}$ potassium hydroxide solution. The organic layers were combined, dried $\left(\mathrm{MgSO}_{4}\right)$, filtered and concentrated in vacuo to afford a crude oil. Purification by flash chromatography (eluting with $80 \%$ ethyl acetate/hexanes doped with $1 \%$ triethylamine, followed by $10 \%$ methanol/ethyl acetate doped with $1 \%$ triethylamine) furnished the diol $(19.1 \mathrm{mg}, 100 \%)$ as a colorless oil $(d s=$ $4: 1)$.

The diol (85.8 mg, $0.26 \mathrm{mmol})$ was dissolved in $\mathrm{CH}_{2} \mathrm{Cl}_{2}(2.6 \mathrm{ml})$ and cooled with stirring to $0{ }^{\circ} \mathrm{C}$ under an atmosphere of nitrogen. Pyridine $(42 \mu \mathrm{L}, 0.52 \mathrm{mmol})$ was added followed by triethylsilyl chloride ( $44 \mu \mathrm{L}, 2.6 \mathrm{mmol}$ ), and the resulting mixture stirred at $0{ }^{\circ} \mathrm{C}$ for $c a .2$ hours (t.1.c. control). 4-(dimethylamino)pyridine $(95.3 \mathrm{mg}, 0.78 \mathrm{mmol})$ was then added followed by acetic anhydride $(38 \mu \mathrm{L}, 0.39 \mathrm{mmol})$, and the reaction stirred for $c a$. 1 hour (t.l.c. control) at 0 ${ }^{\circ} \mathrm{C}$. The reaction was absorbed on to silica gel column and purified by flash chromatography (eluting with $15-20 \%$ ethyl acetate/hexanes to furnish $\mathbf{8 a} / \mathbf{b}(89.8 \mathrm{mg}, 71 \%)$ as a colorless oil ( $d s$ =4:1). The diastereoisomers were then separated using a semi-preparative HPLC using a HPZorbax RX-SIL semi-preparative (9.4 x $250 \mathrm{~mm}$ 5-micron) column eluting with 1\% 2propanol/hexanes at $2 \mathrm{ml} / \mathrm{min}$ at $20{ }^{\circ} \mathrm{C}\left(\mathrm{t}_{\mathrm{R}}(\mathbf{8 b})=17.2 \mathrm{~min} . / \mathrm{t}_{\mathrm{R}}(\mathbf{8 a})=18.5 \mathrm{~min}\right.$. $):[\alpha]_{\mathrm{D}}{ }^{23}+4.0(\mathrm{c}=$ 1.06, $\mathrm{CHCl}_{3}$ ); IR (neat) 3011 (m), 2957 (s), 2913 (s), 2877 (s), 2839 (m), 1738 (s), 1678 (s), 
1602 (s), 1576 (m), 1513 (s), 1464 (m) cm ${ }^{-1}$; ${ }^{1} \mathrm{H}$ NMR (400 MHz, $\left.\mathrm{CDCl}_{3}\right) \delta 7.86$ (d, J=8.7 Hz, 2H), $7.17(\mathrm{~d}, J=8.5 \mathrm{~Hz}, 2 \mathrm{H}), 6.87(\mathrm{~d}, J=8.9 \mathrm{~Hz}, 2 \mathrm{H}), 6.77$ (d, $J=8.6 \mathrm{~Hz}, 2 \mathrm{H}), 5.16$ (q, $J=5.4$ $\mathrm{Hz}, 1 \mathrm{H}), 3.83(\mathrm{~s}, 3 \mathrm{H}), 3.78(\mathrm{dt}, J=8.3,5.5 \mathrm{~Hz}, 1 \mathrm{H}), 3.74(\mathrm{~s}, 3 \mathrm{H}), 3.58\left(\mathrm{dd}, \mathrm{A}\right.$ of ABX, $J_{A B}=10.9$ $\left.\mathrm{Hz}, J_{A X}=5.8 \mathrm{~Hz}, 1 \mathrm{H}\right), 3.50\left(\mathrm{dd}, \mathrm{B}\right.$ of $\left.\mathrm{ABX}, J_{A B}=10.8 \mathrm{~Hz}, J_{B X}=5.2 \mathrm{~Hz}, 1 \mathrm{H}\right), 3.35(\mathrm{dd}, \mathrm{A}$ of $\left.\mathrm{ABX}, J_{A B}=16.8 \mathrm{~Hz}, J_{A X}=5.7 \mathrm{~Hz}, 1 \mathrm{H}\right), 3.28\left(\mathrm{dd}, \mathrm{B}\right.$ of $\left.\mathrm{ABX}, J_{A B}=16.6 \mathrm{~Hz}, J_{B X}=8.7 \mathrm{~Hz}, 1 \mathrm{H}\right)$ $1.97(\mathrm{~s}, 3 \mathrm{H}), 0.90(\mathrm{t}, J=7.9 \mathrm{~Hz}, 9 \mathrm{H}), 0.54(\mathrm{q}, J=7.9 \mathrm{~Hz}, 6 \mathrm{H}) ;{ }^{13} \mathrm{C} \mathrm{NMR}\left(100 \mathrm{MHz}, \mathrm{CDCl}_{3}\right) \delta$ 196.77 (e), 170.67 (e), 163.56 (e), 158.51 (e), 132.16 (e), 130.53 (o), 130.32 (e), 130.01 (o), 113.83 (o) 113.75 (o), 76.74 (o), 62.52 (e), 55.65 (o) 55.36 (o), 41.06 (o) 40.93 (e), 21.26 (o), 6.92 (o), 4.54 (e); HRMS (CI, $\mathrm{M}+\mathrm{H}^{+}$) calcd for $\mathrm{C}_{27} \mathrm{H}_{39} \mathrm{O}_{6} \mathrm{Si} 487.2516$, found 487.2537.

(-)-Sugiresinol Dimethyl Ether 9. The ketone 8a $(17.6 \mathrm{mg}, 0.036 \mathrm{mmol})$ was dissolved in acetonitrile $(360 \mu \mathrm{L})$ and stirred at room temperature under an atmosphere of nitrogen. Bismuth(III) bromide $(4.8 \mathrm{mg}, 0.011 \mathrm{mmol})$ was added followed by triethylsilane $(17 \mu \mathrm{L}, 0.110$ $\mathrm{mmol}$ ), and the resulting reaction mixture stirred for $c a .45$ minutes (t.l.c. control). The reaction mixture was then cooled to $0{ }^{\circ} \mathrm{C}$ and methanol $(150 \mu \mathrm{L})$ followed by sodium tert-butoxide $(38.3$ $\mathrm{mg}, 0.399 \mathrm{mmol}$ ) were added, and the resulting reaction stirred at $0{ }^{\circ} \mathrm{C}$ for $\mathrm{ca} 2$ hours (t.l.c. control). The reaction mixture was then absorbed on to silica gel column and purified by flash chromatography (eluting with 30-35\% ethyl acetate/hexanes) to afford $9(11.3 \mathrm{mg}, 99 \%)$ as a white crystalline solid: $[\alpha]_{\mathrm{D}}{ }^{26}-3.9\left(\mathrm{c}=0.67, \mathrm{CHCl}_{3}\right)$; IR (neat) $3377(\mathrm{bs}), 3009(\mathrm{~m}), 2920(\mathrm{~m})$, 2838 (m), 1612 (m), 1585 (m), 1515 (s), 1463 (m), 1443 (m) cm ${ }^{-1} ;{ }^{1} \mathrm{H}$ NMR (500 MHz, CDCl $\left.{ }_{3}\right)$ $\delta 7.28(\mathrm{dt}, J=8.6,2.8 \mathrm{~Hz}, 2 \mathrm{H}), 7.21(\mathrm{dt}, J=8.8,2.4 \mathrm{~Hz}, 2 \mathrm{H}), 6.88(\mathrm{dt}, J=8.8,2.3 \mathrm{~Hz}, 2 \mathrm{H}), 6.85$ $(\mathrm{dt}, J=8.8,2.4 \mathrm{~Hz}, 2 \mathrm{H}), 4.46(\mathrm{dd}, J=11.2,1.9 \mathrm{~Hz}, 1 \mathrm{H}), 4.28(\mathrm{dd}, J=11.0,4.9 \mathrm{~Hz}, 1 \mathrm{H}), 3.84$ (dddd, $J=9.9,7.7,4.8,3.2 \mathrm{~Hz} 1 \mathrm{H}), 3.78(\mathrm{~s}, 3 \mathrm{H}), 3.77(\mathrm{~s}, 3 \mathrm{H}), 3.47$ (dd, $J=11.0,10.1 \mathrm{~Hz}, 1 \mathrm{H})$, $2.76(\mathrm{ddd}, J=12.8,9.7,3.5 \mathrm{~Hz} 1 \mathrm{H}), 2.05(\mathrm{dt}, J=13.7,3.9,2.3 \mathrm{~Hz}, 1 \mathrm{H}), 1.90$ (ddd, $J=13.7$, 12.4, $11.2 \mathrm{~Hz}, 1 \mathrm{H}), 1.56$ (d, $J=3.0 \mathrm{~Hz}, 1 \mathrm{H}) ;{ }^{13} \mathrm{C} \mathrm{NMR}\left(100 \mathrm{MHz}, \mathrm{CDCl}_{3}\right) \delta 159.25(\mathrm{e}), 158.98$ (e), 134.05 (e), 133.27 (e), 128.99 (o), 127.30 (o), 114.51 (o), 113.95 (o), 79.68 (o) 72.48 (e), 70.85 (o), 55.49 (o), 50.30 (o) 40.56 (e); HRMS (CI, $\mathrm{M}+\mathrm{H}^{+}$) calcd for $\mathrm{C}_{19} \mathrm{H}_{22} \mathrm{O}_{4} 314.1518$, found 314.1514 . 


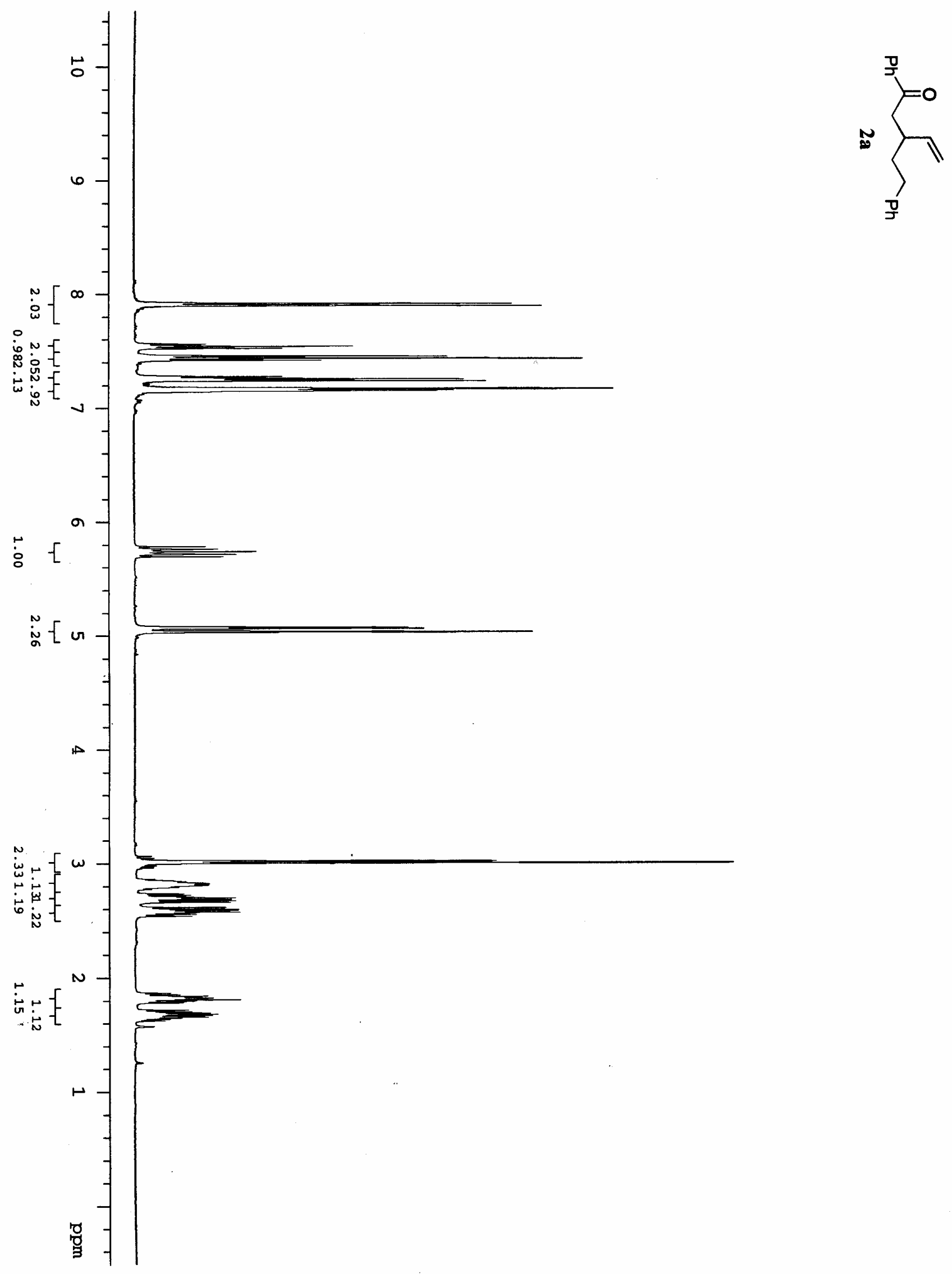

S11 

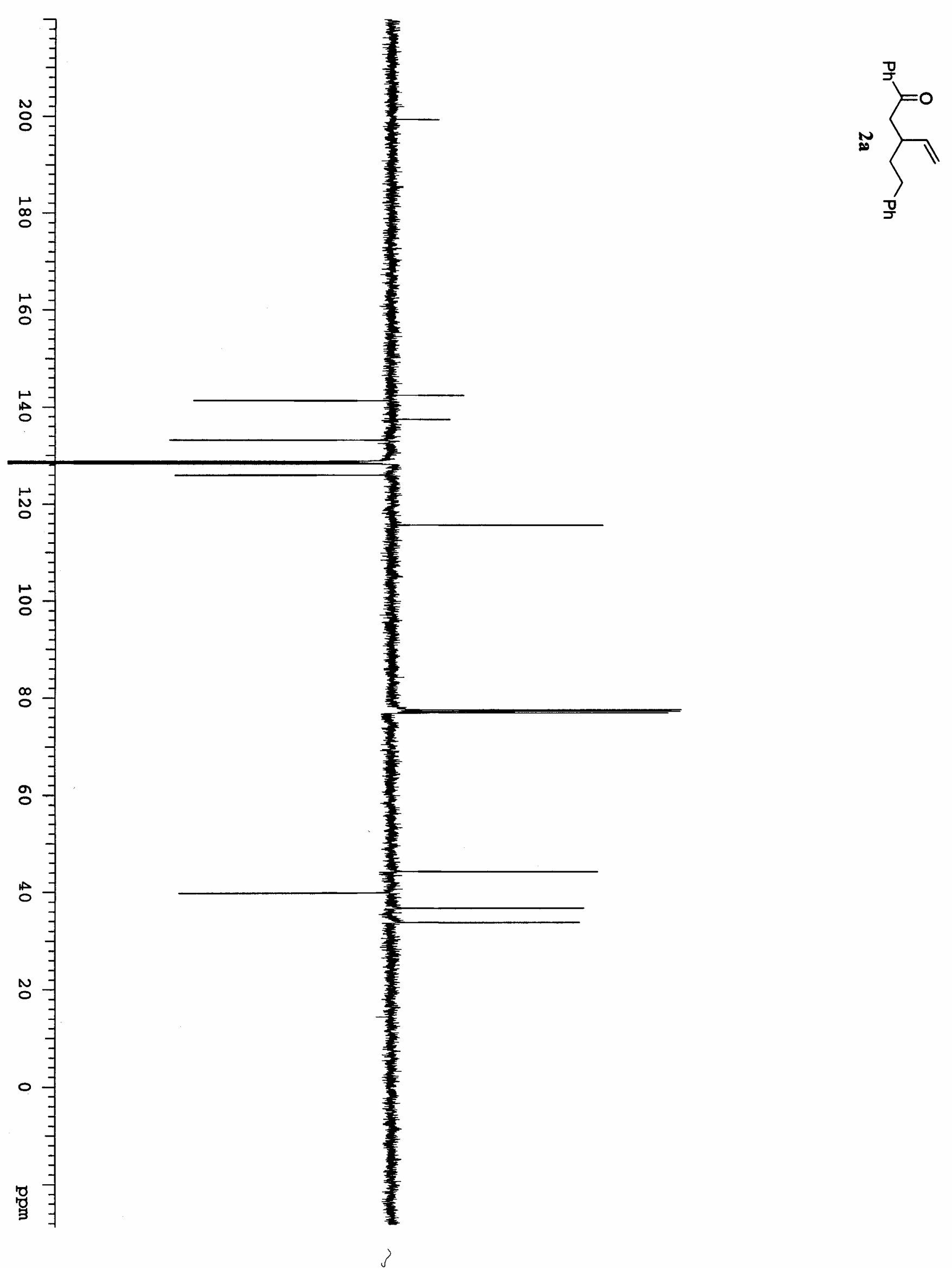


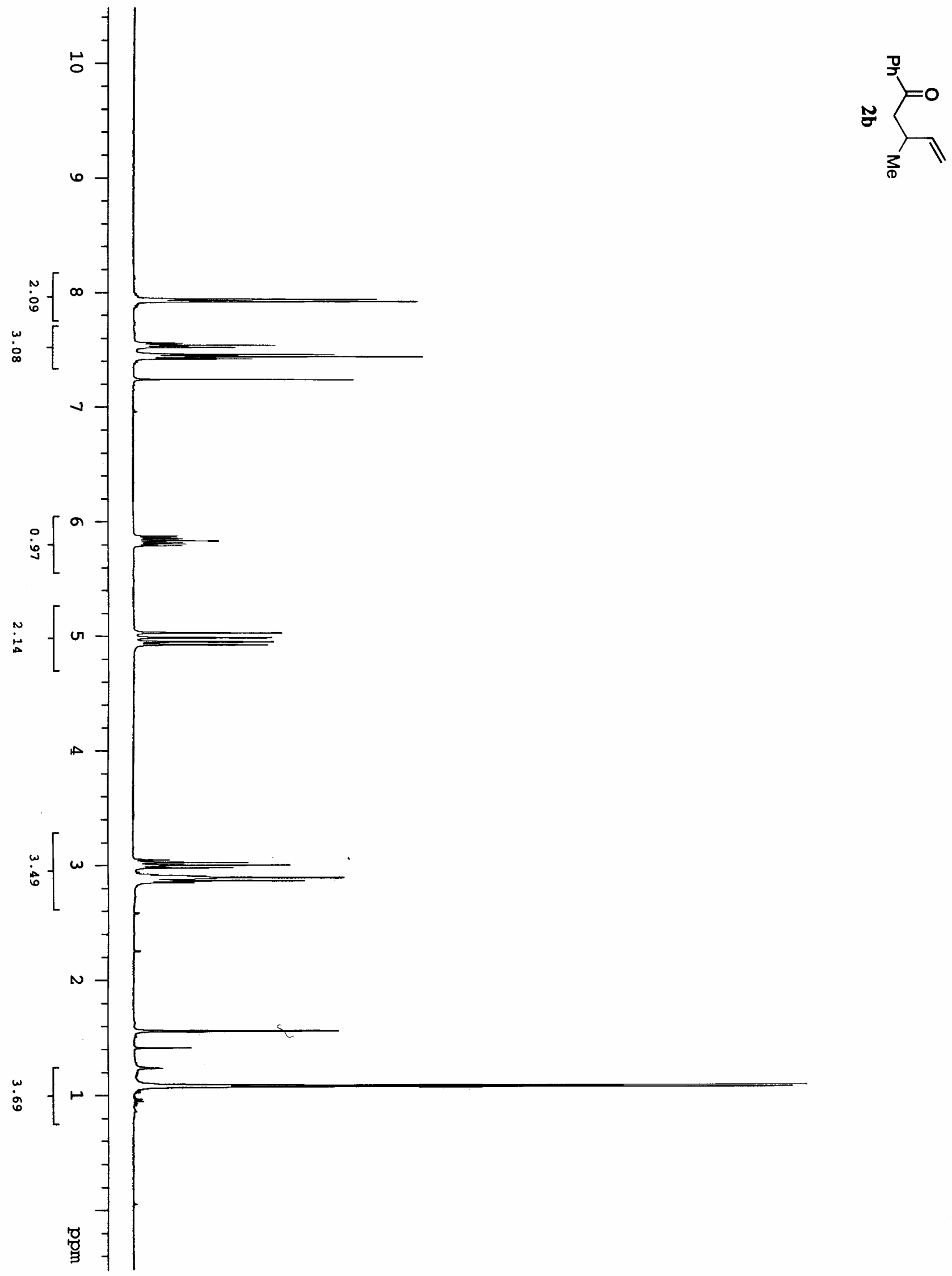




$$
\mid
$$




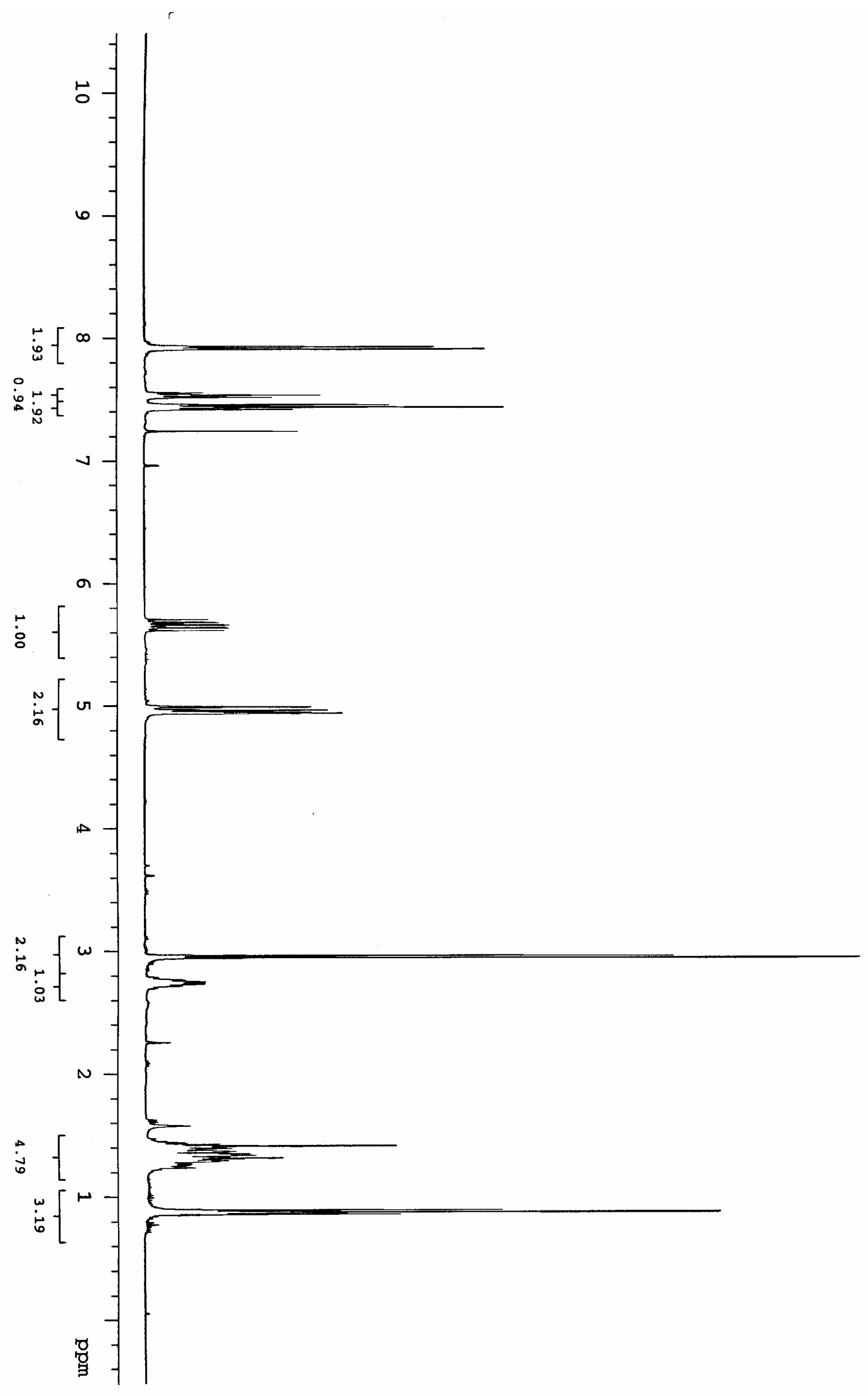




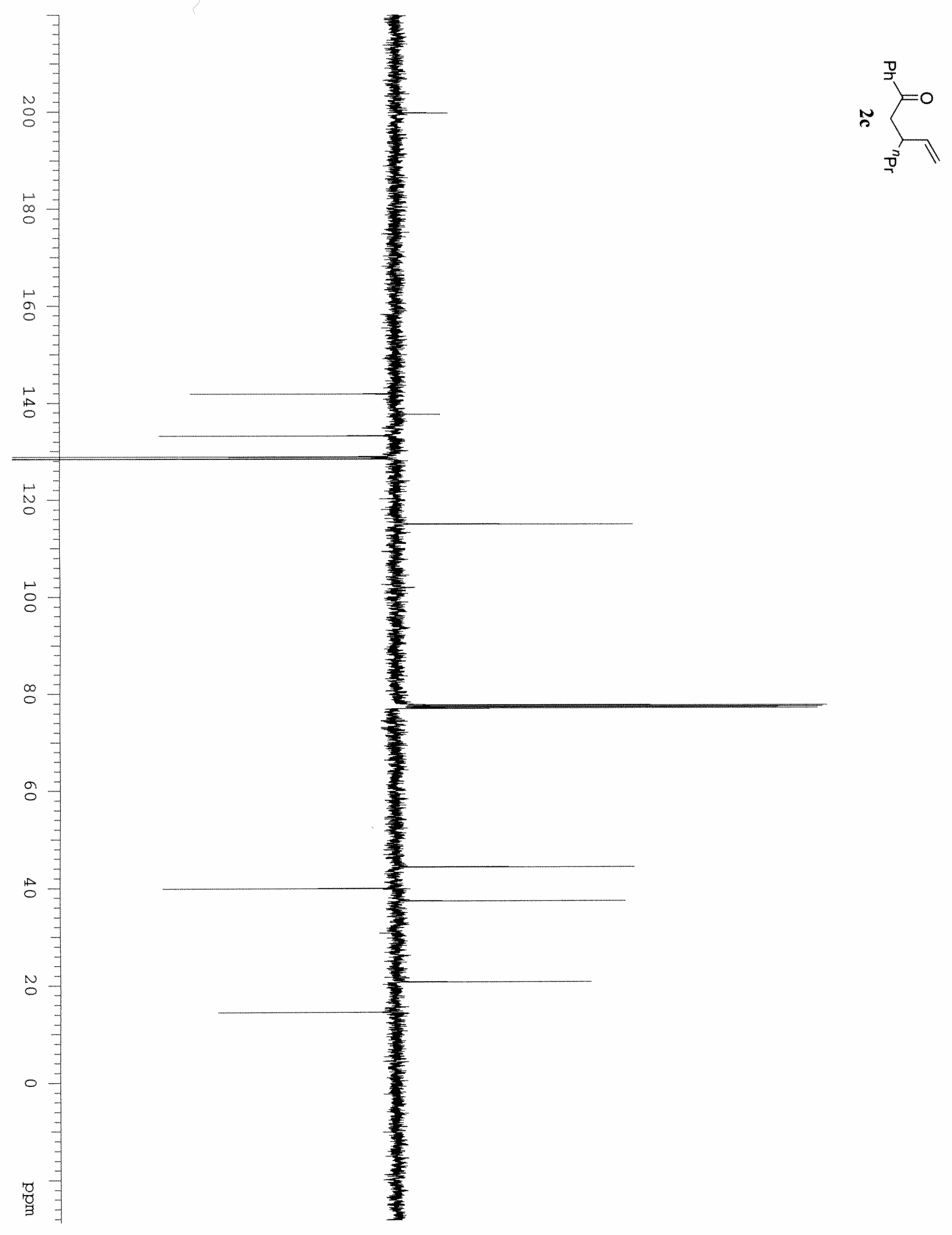



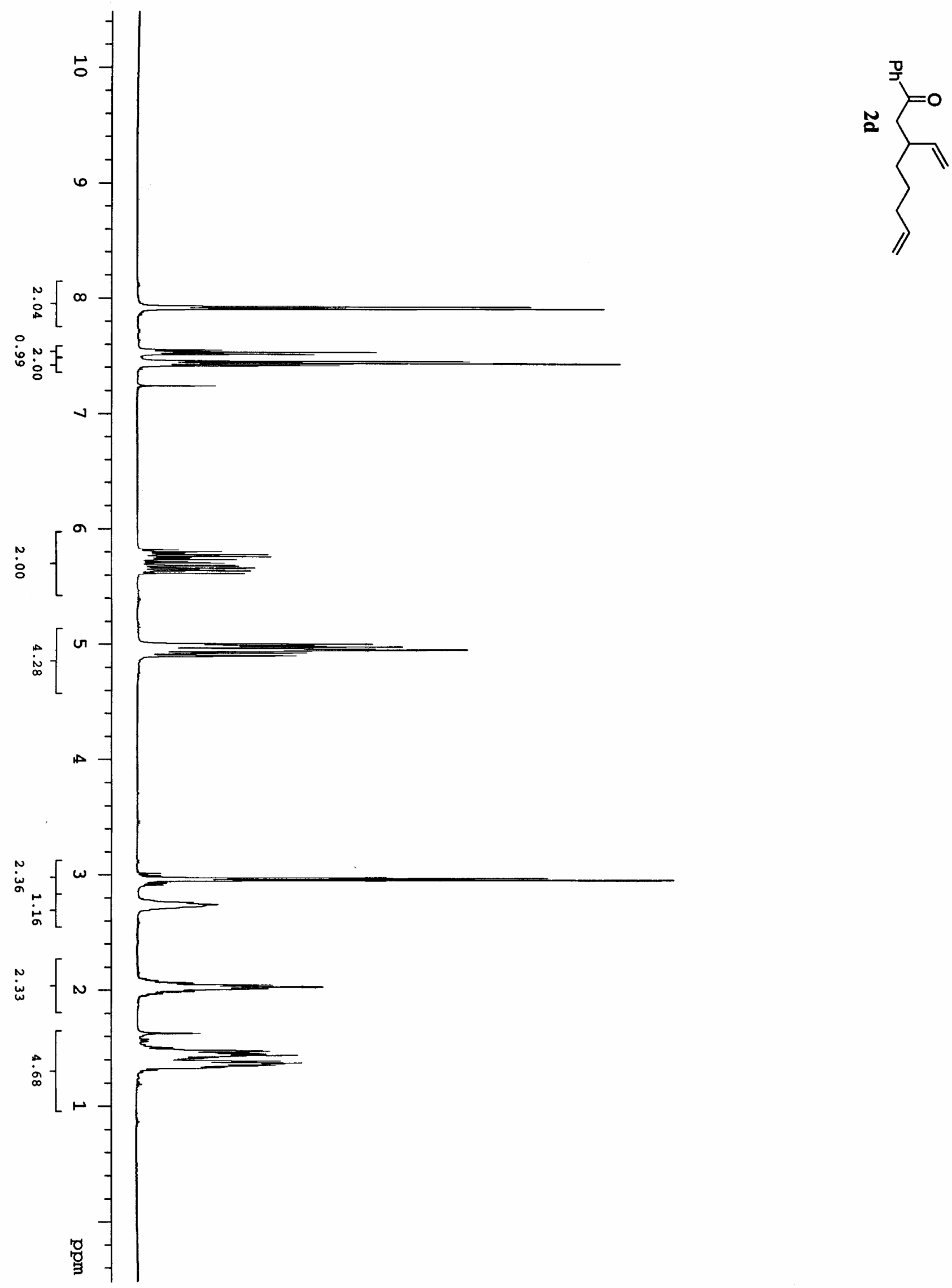

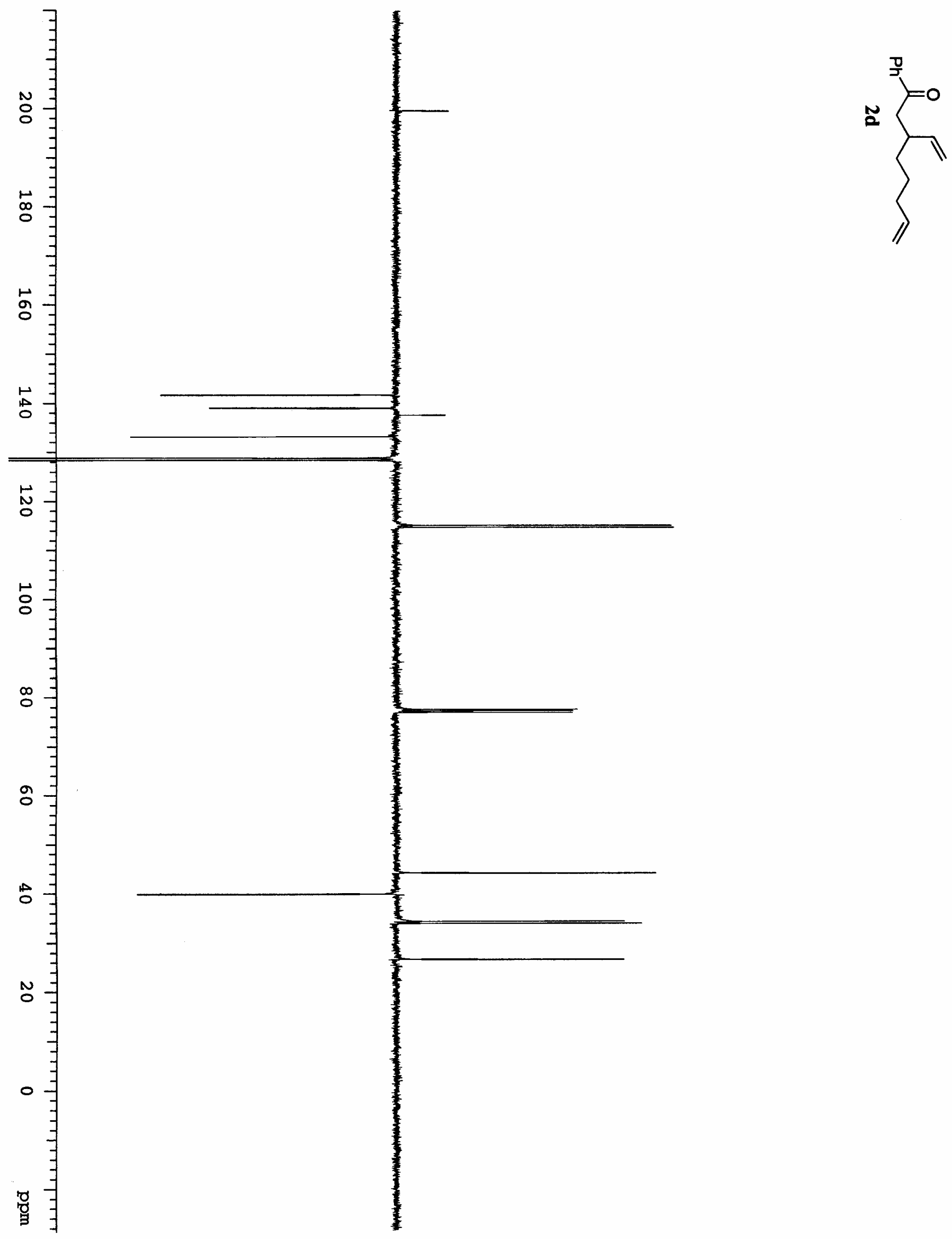

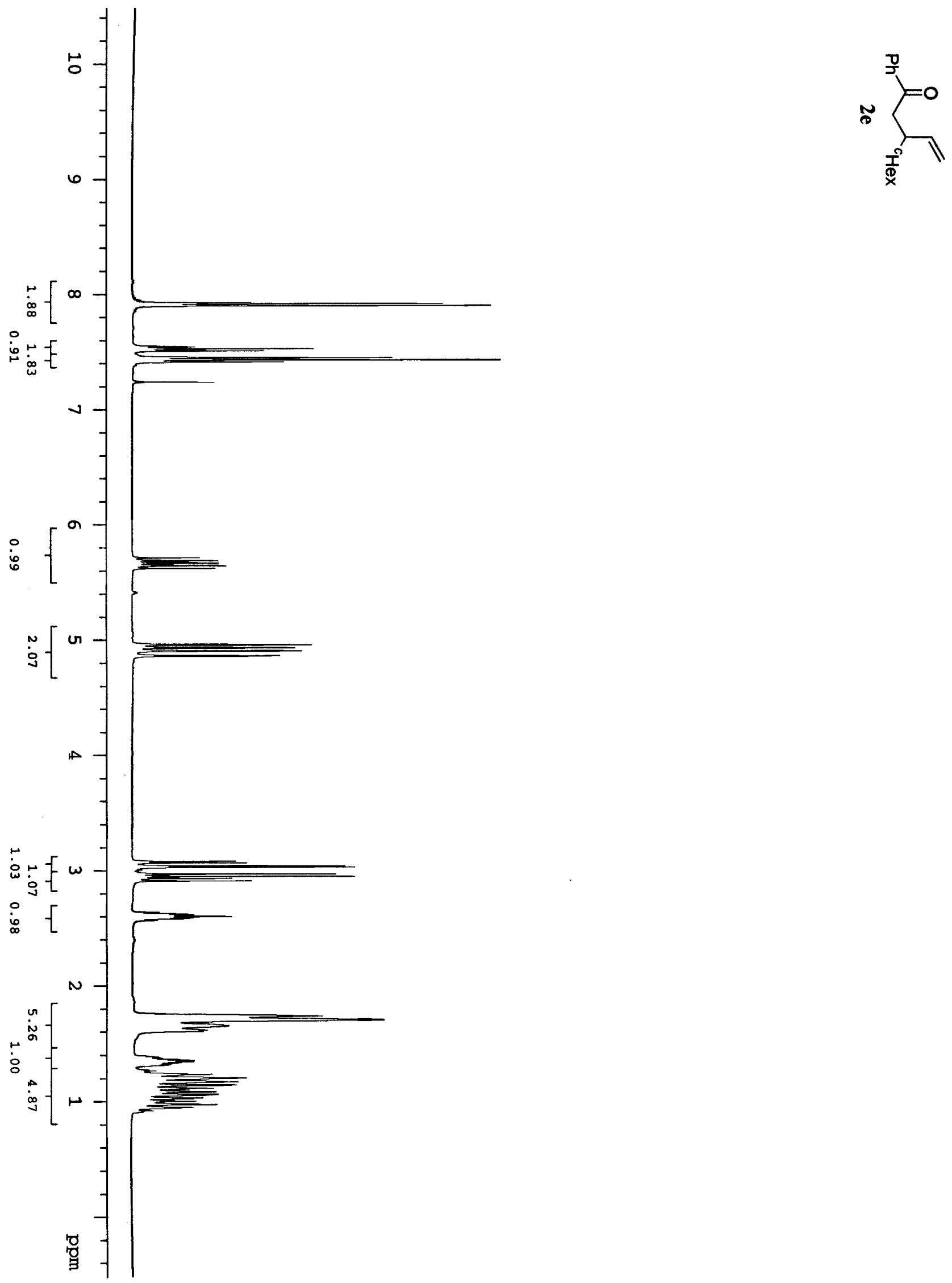


$$
\text { 㭅 }
$$




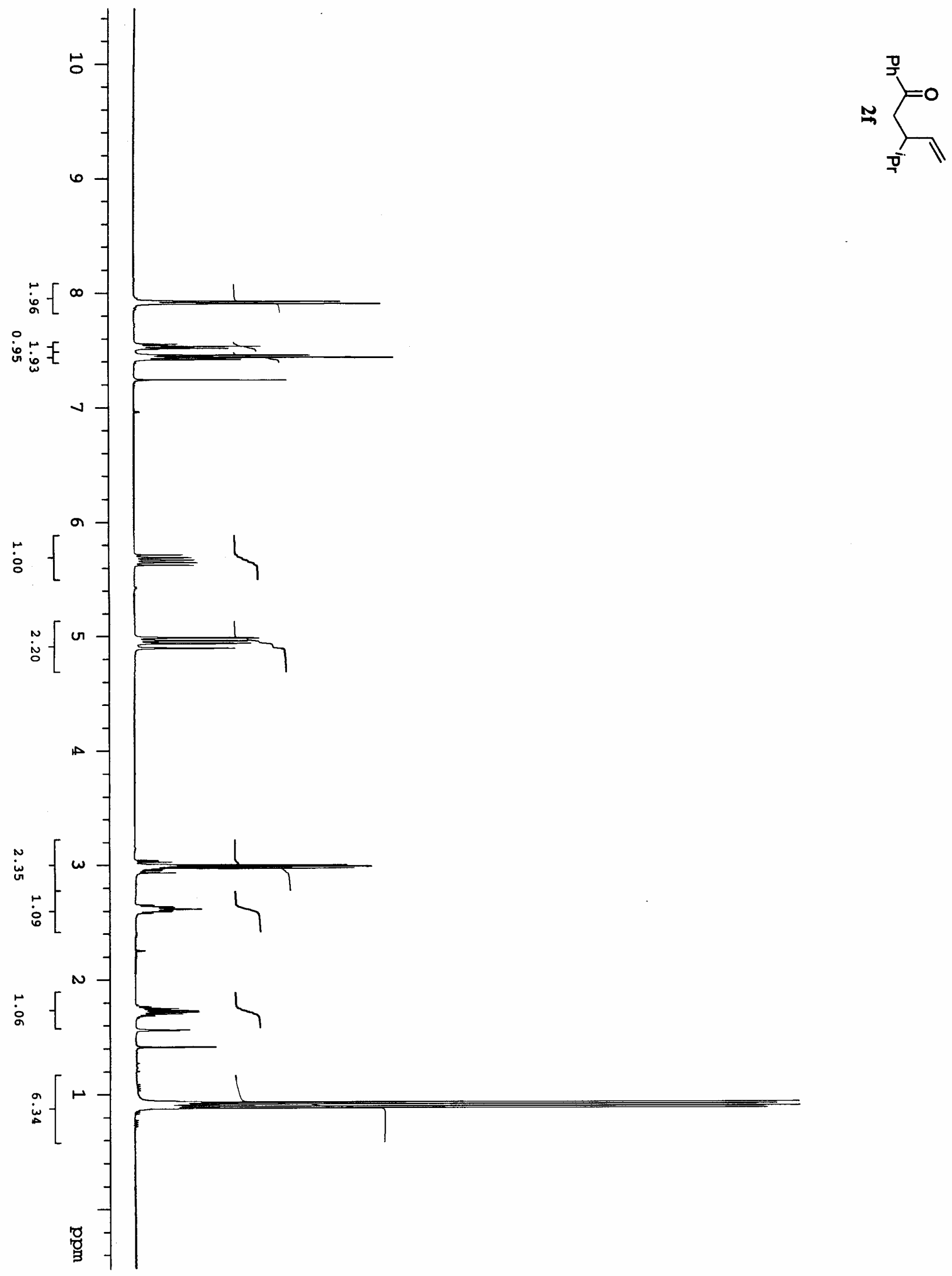

S21 


$$
H
$$




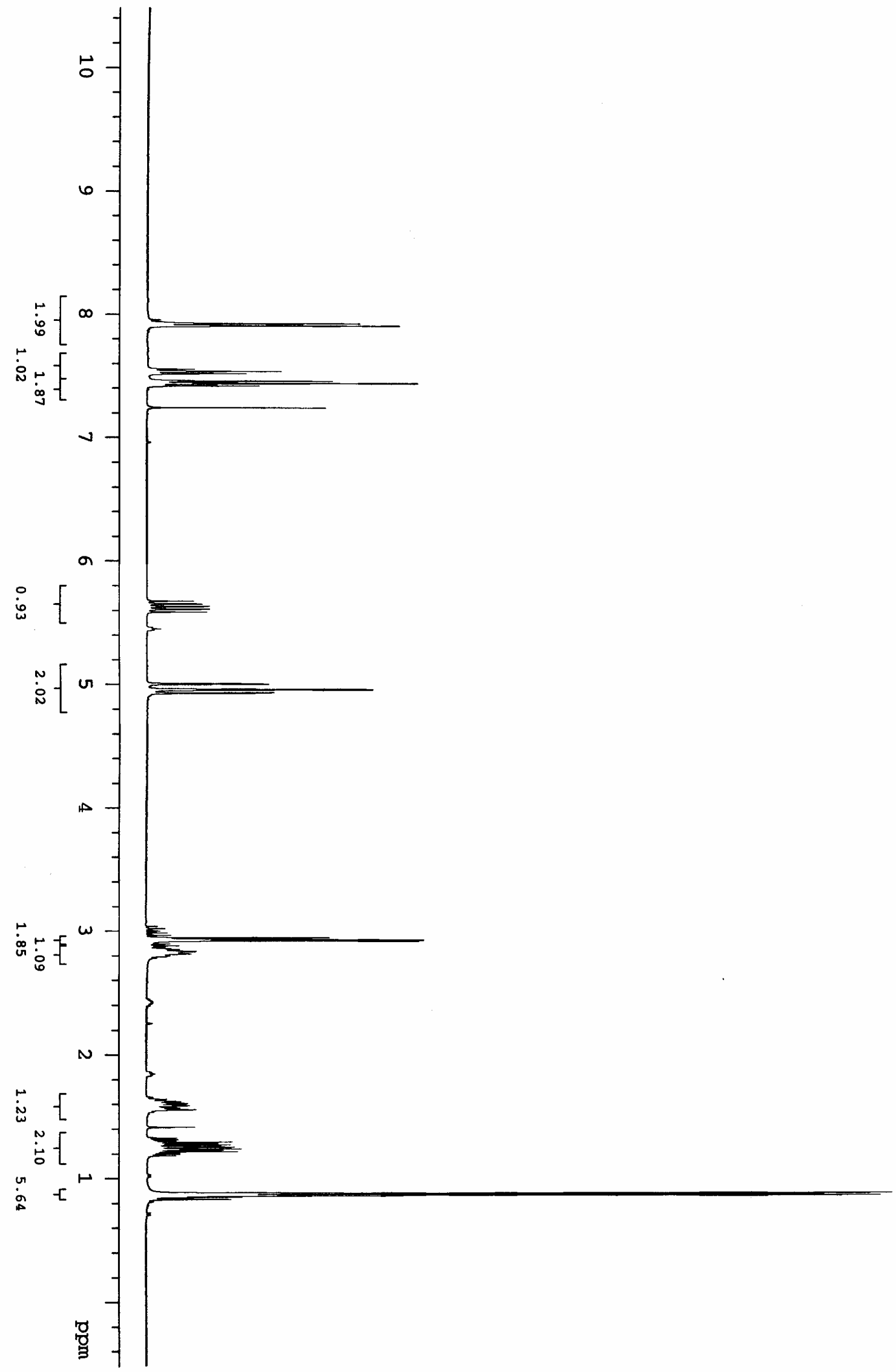




$$
f
$$



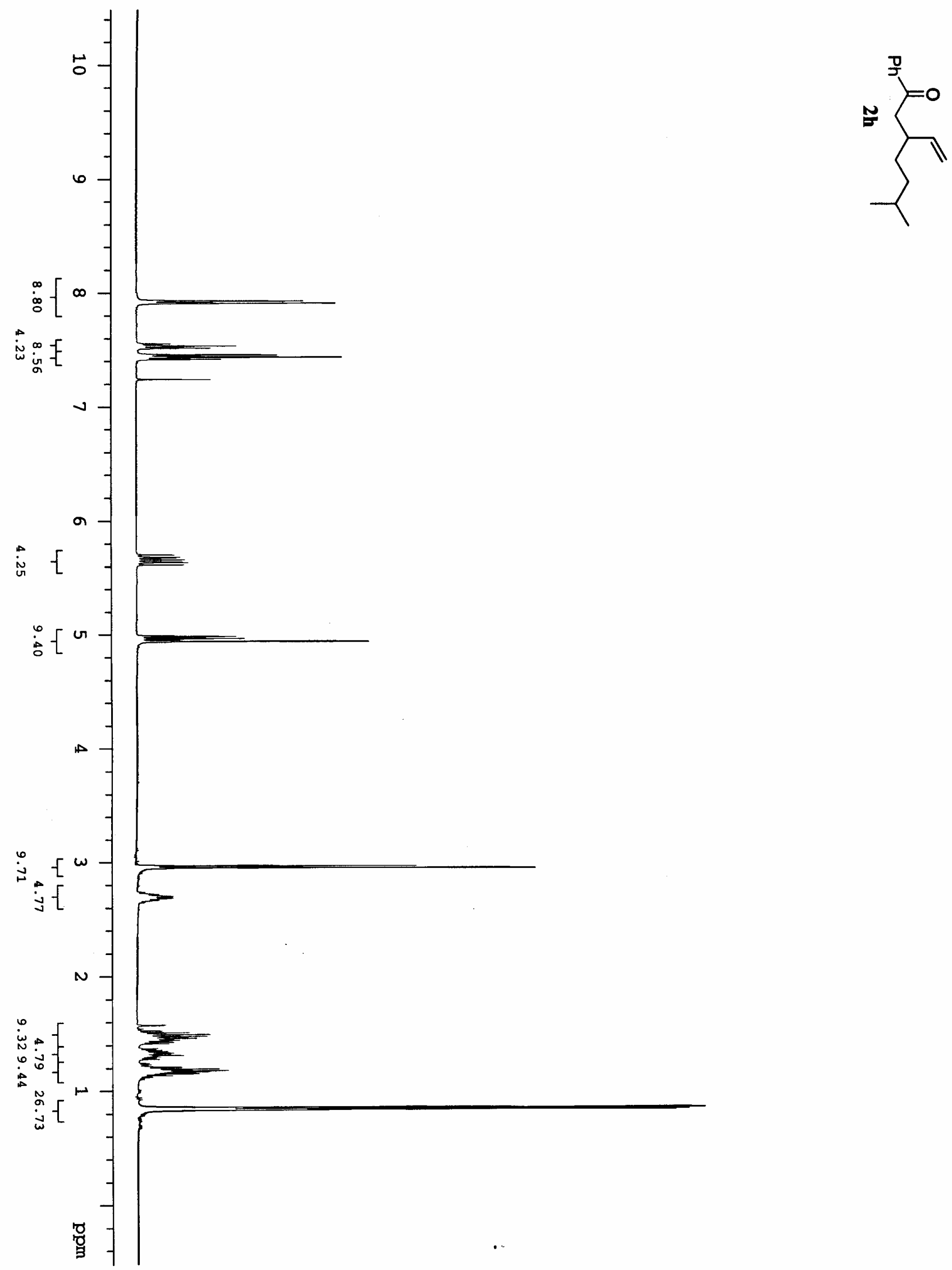


$$
H^{\prime}
$$




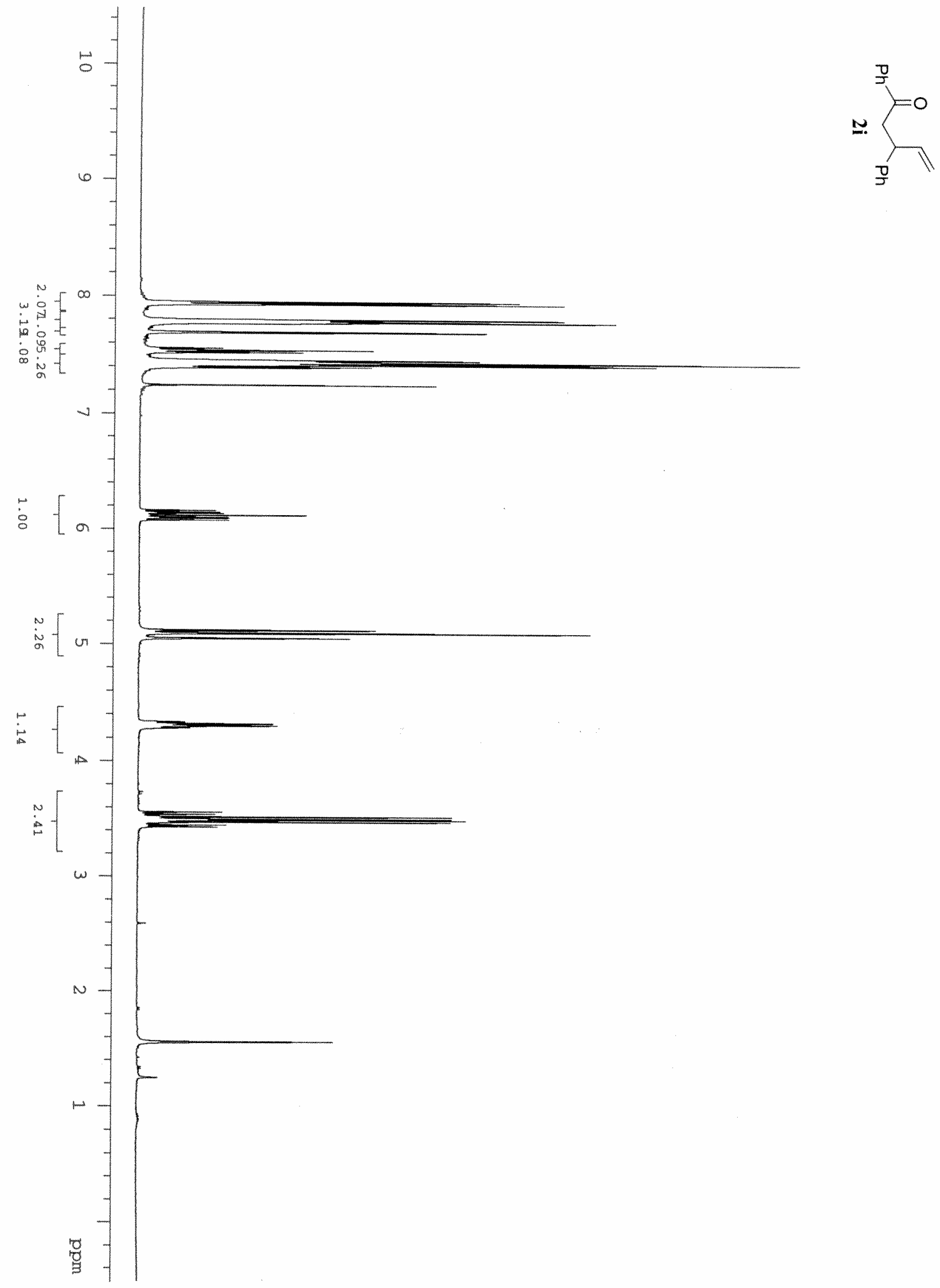




$$
H
$$




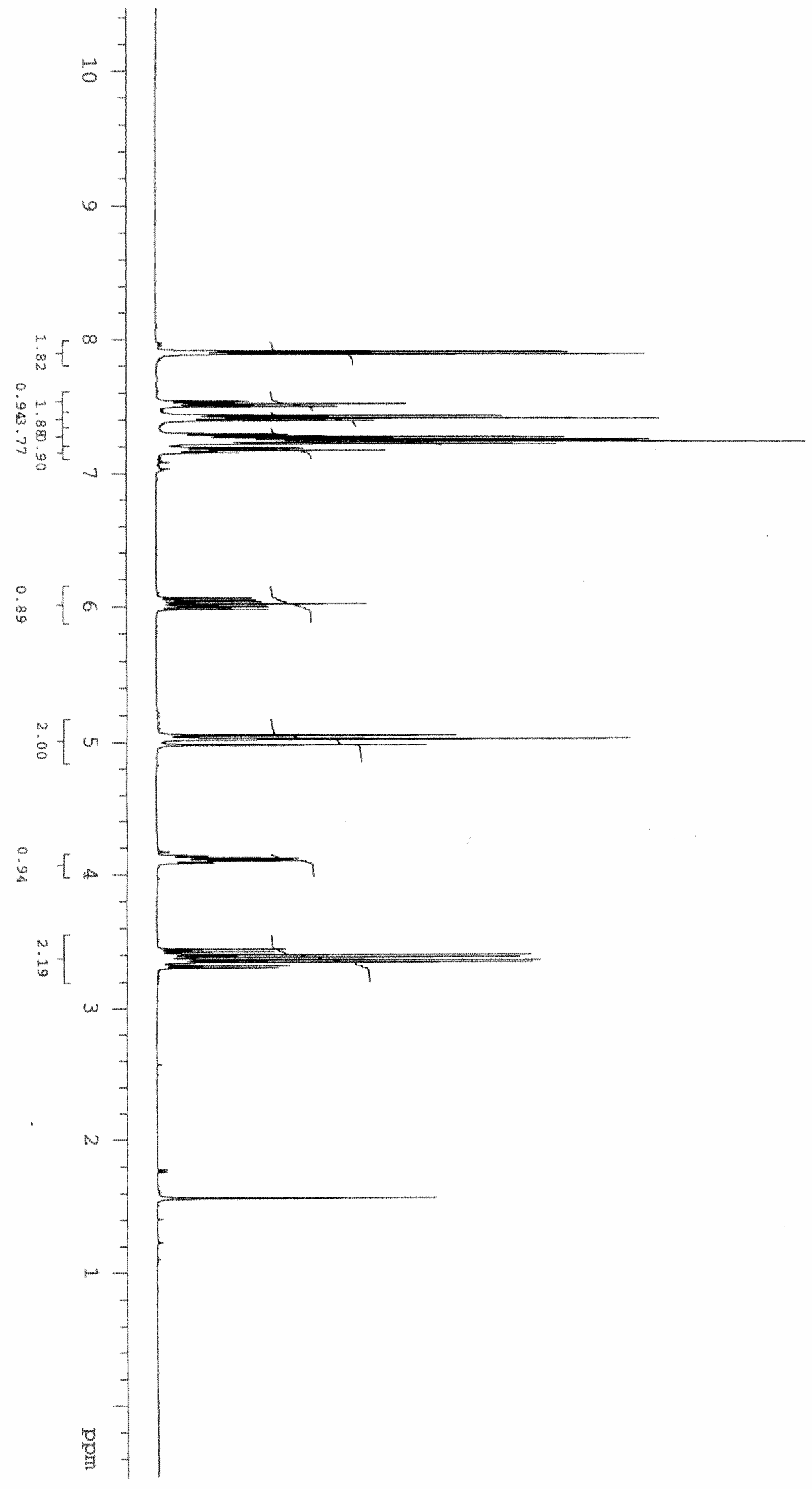




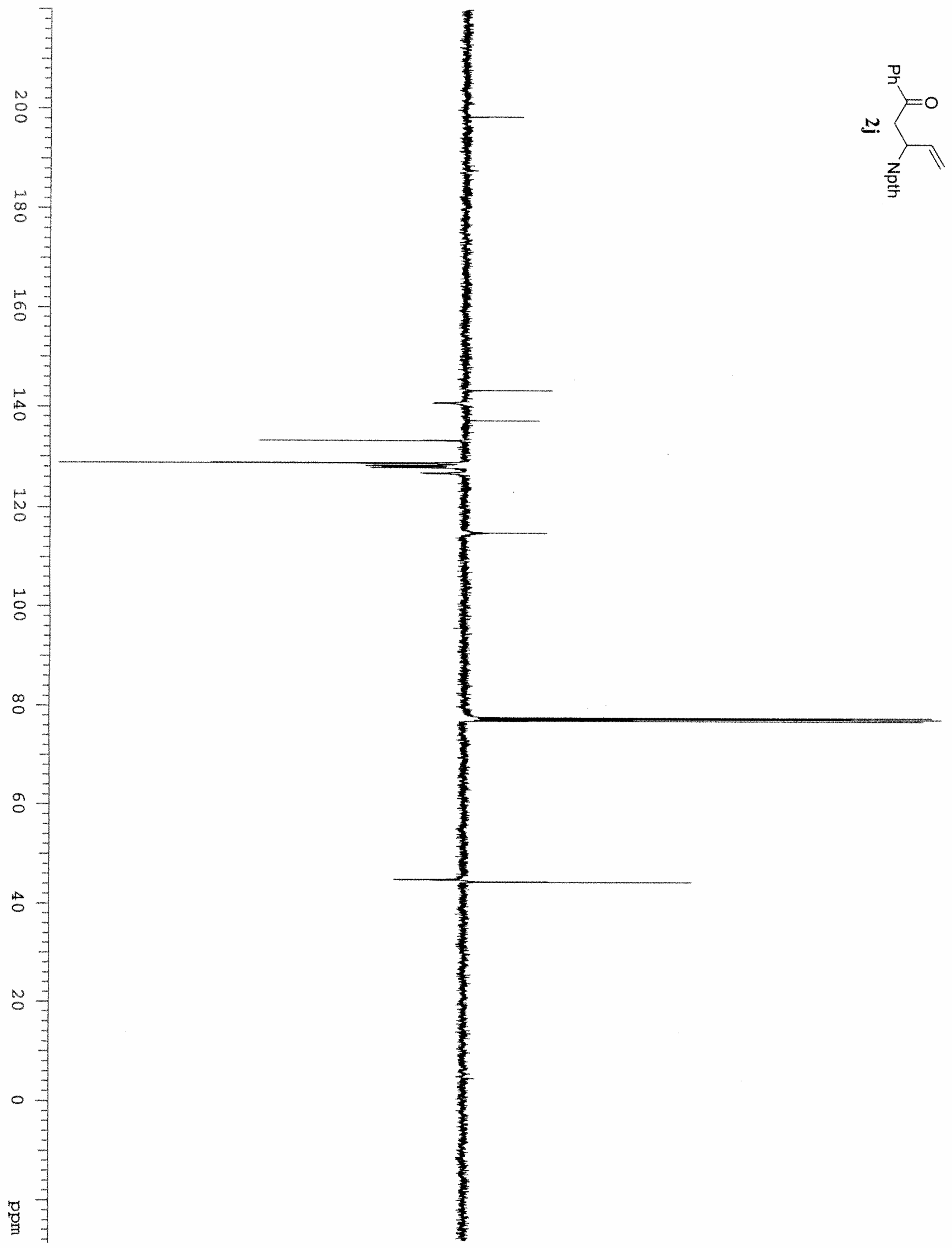

S30 


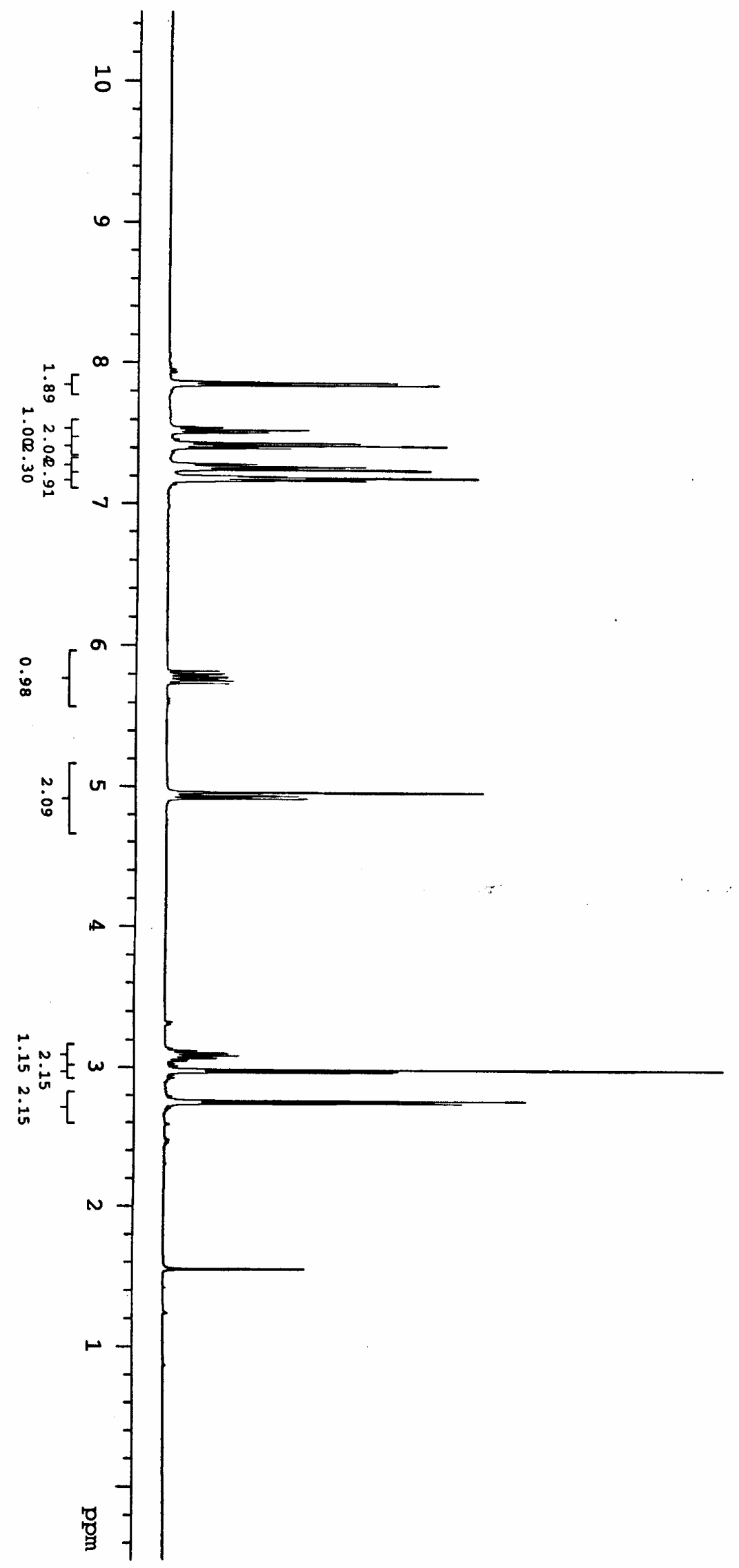




$$
\text { H }
$$



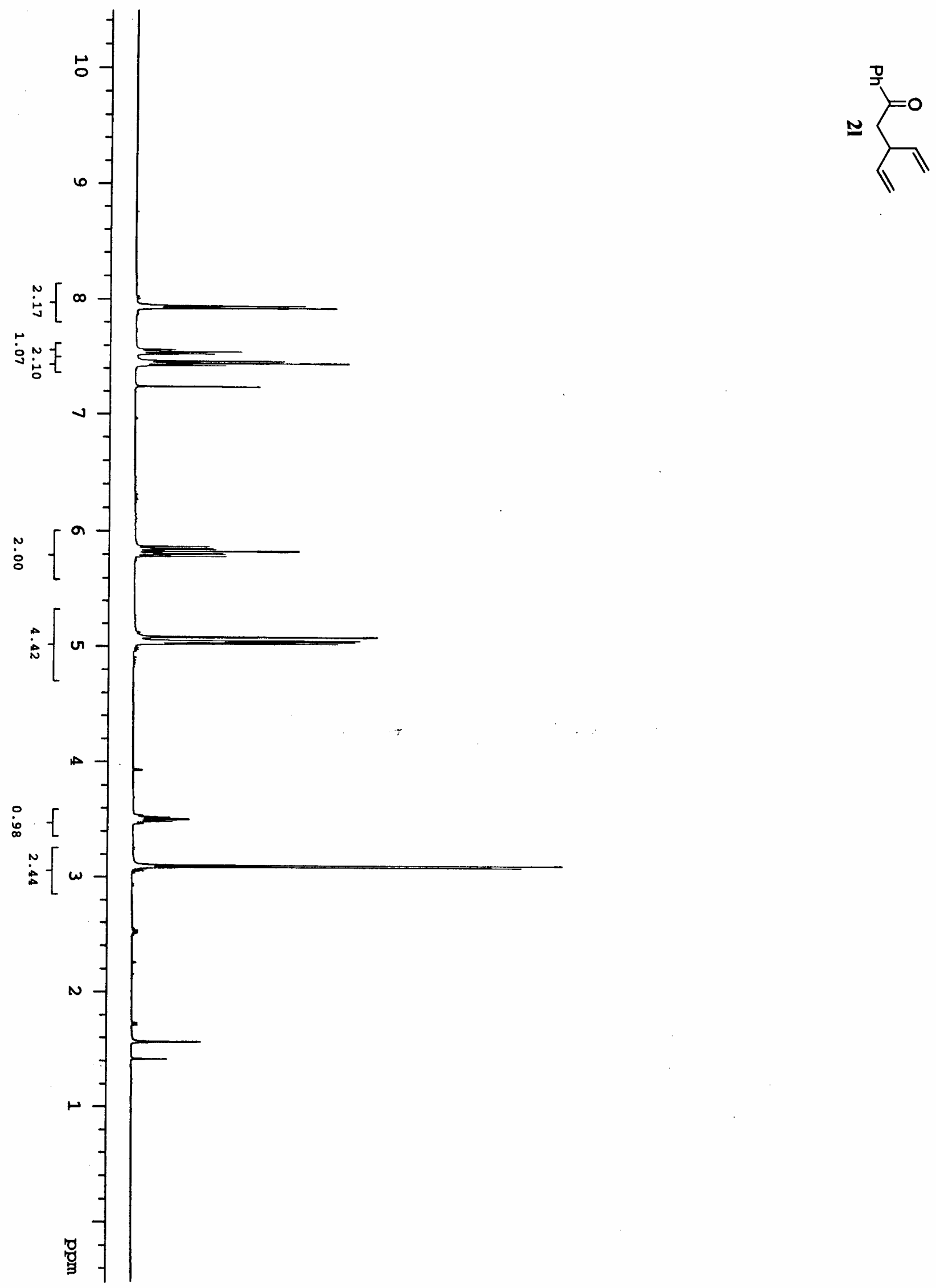


$$
H
$$




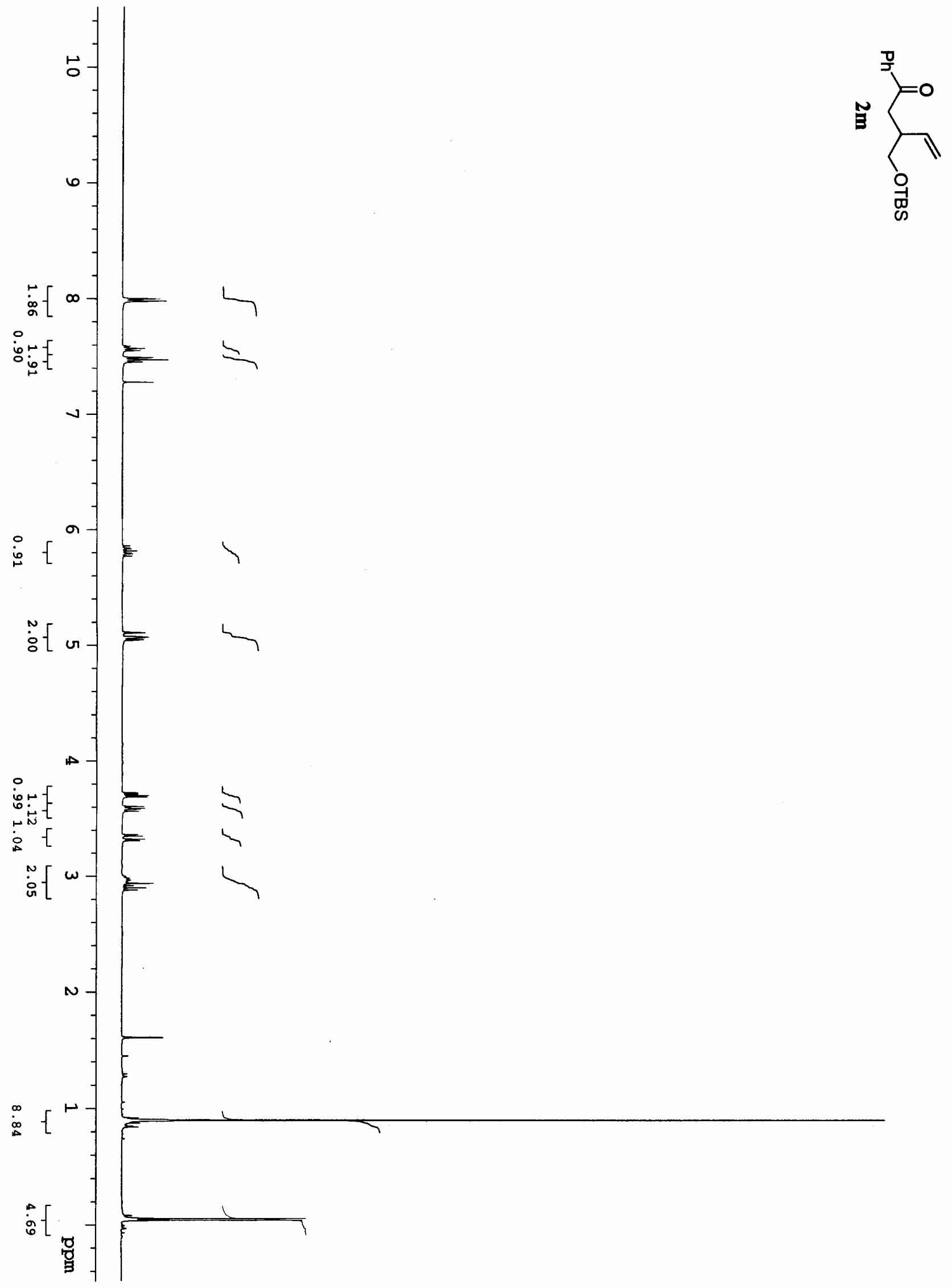




$$
H
$$




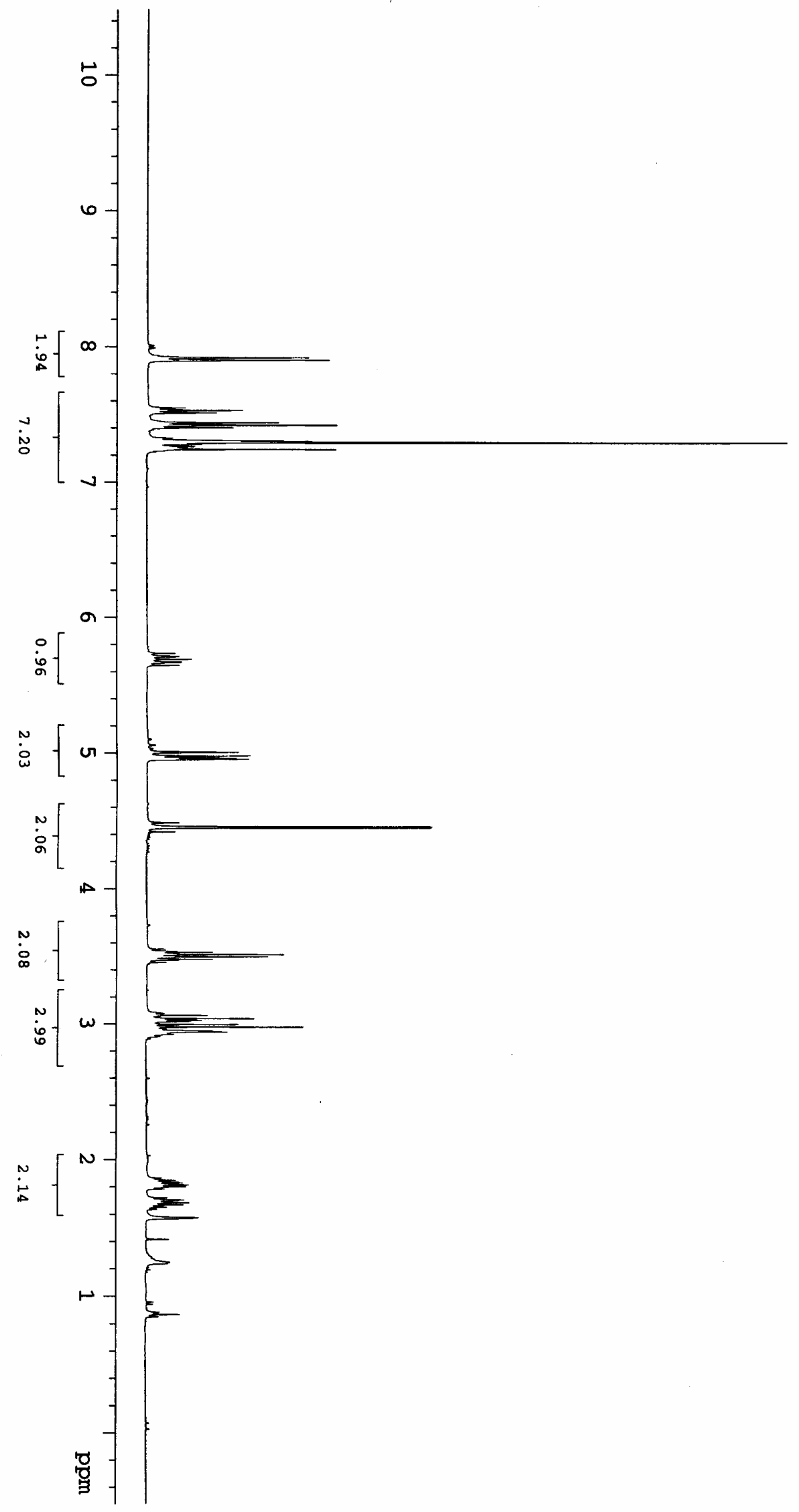




$$
\mid
$$




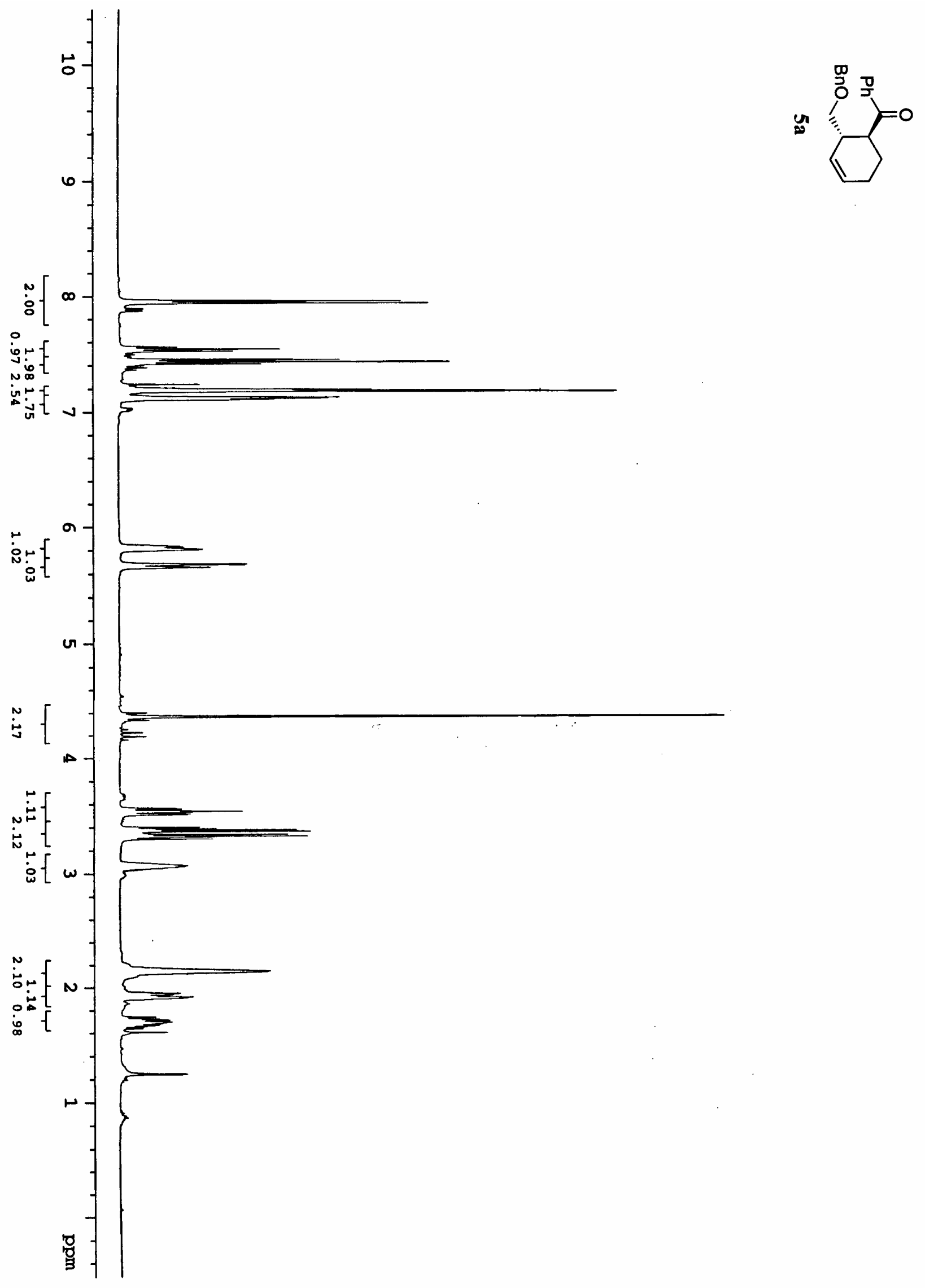




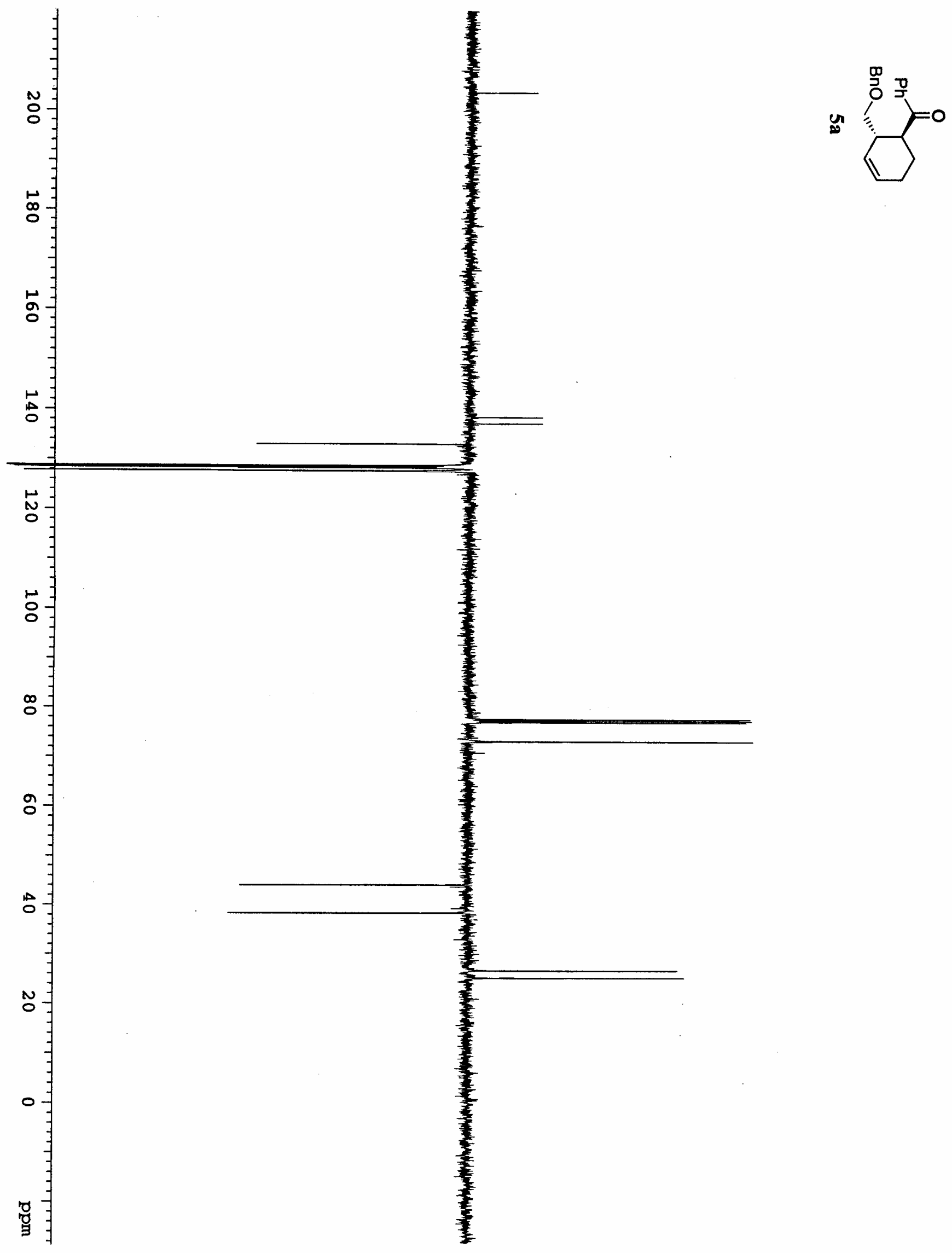

S40 


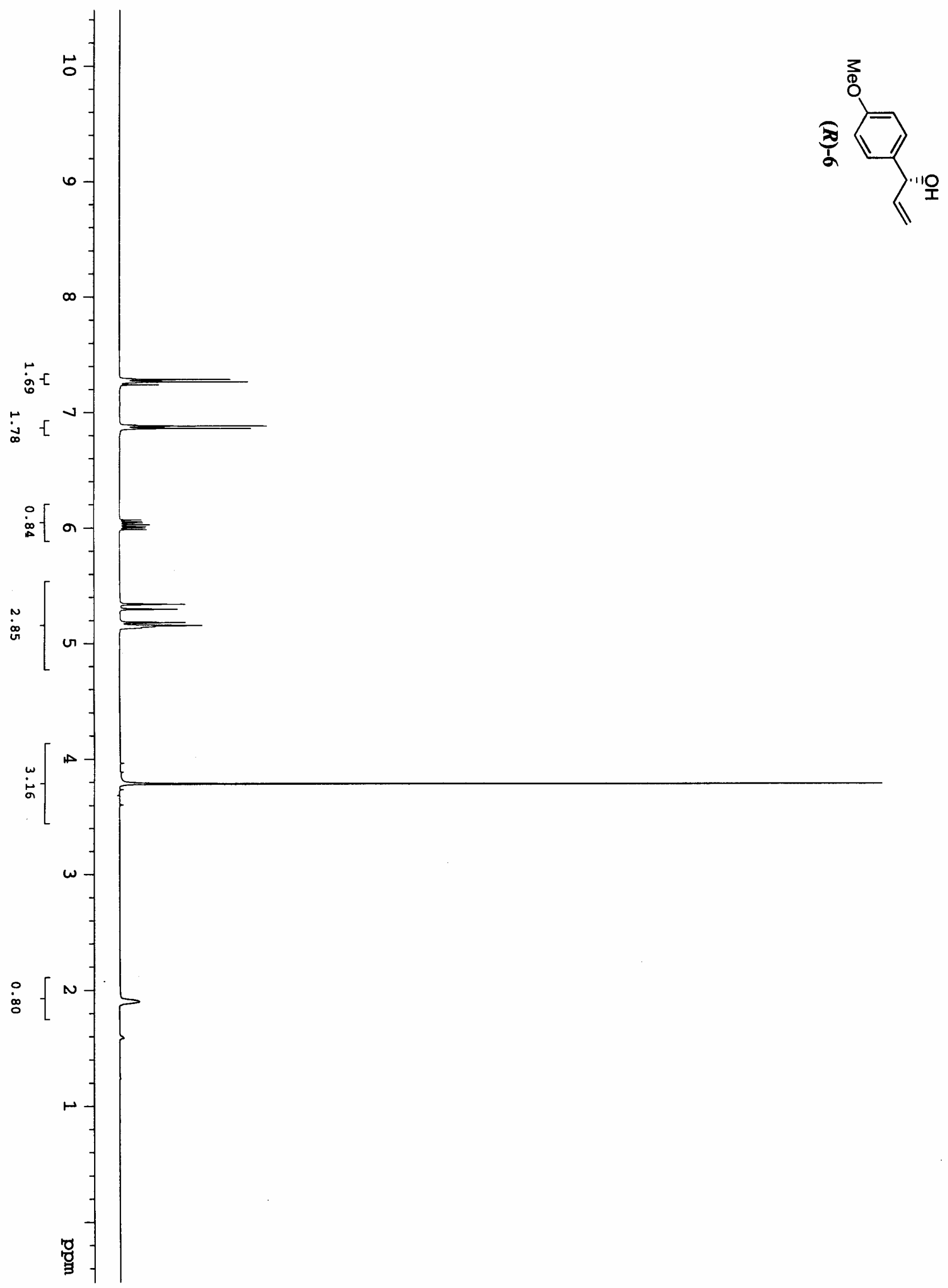




$$
H
$$




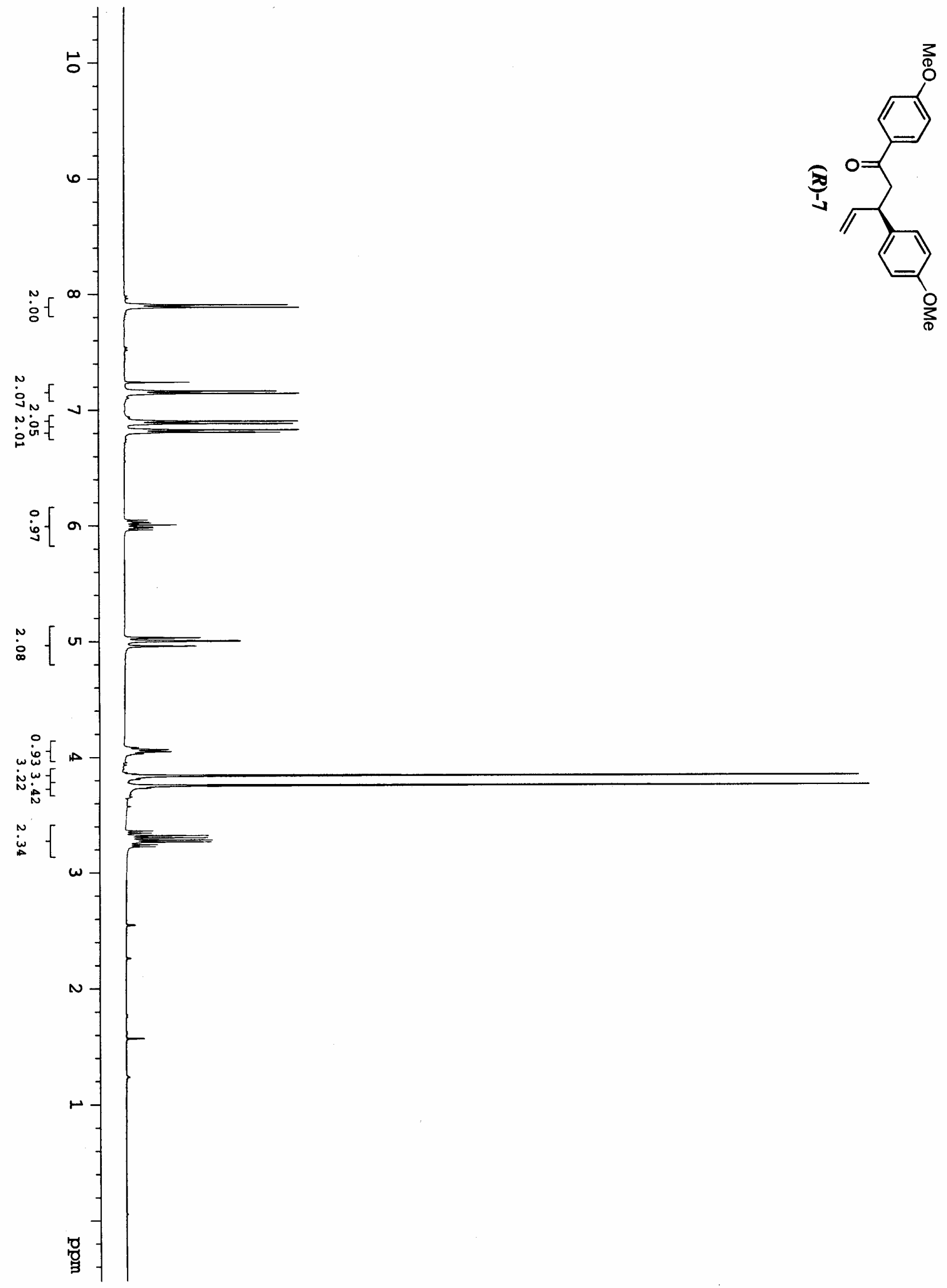



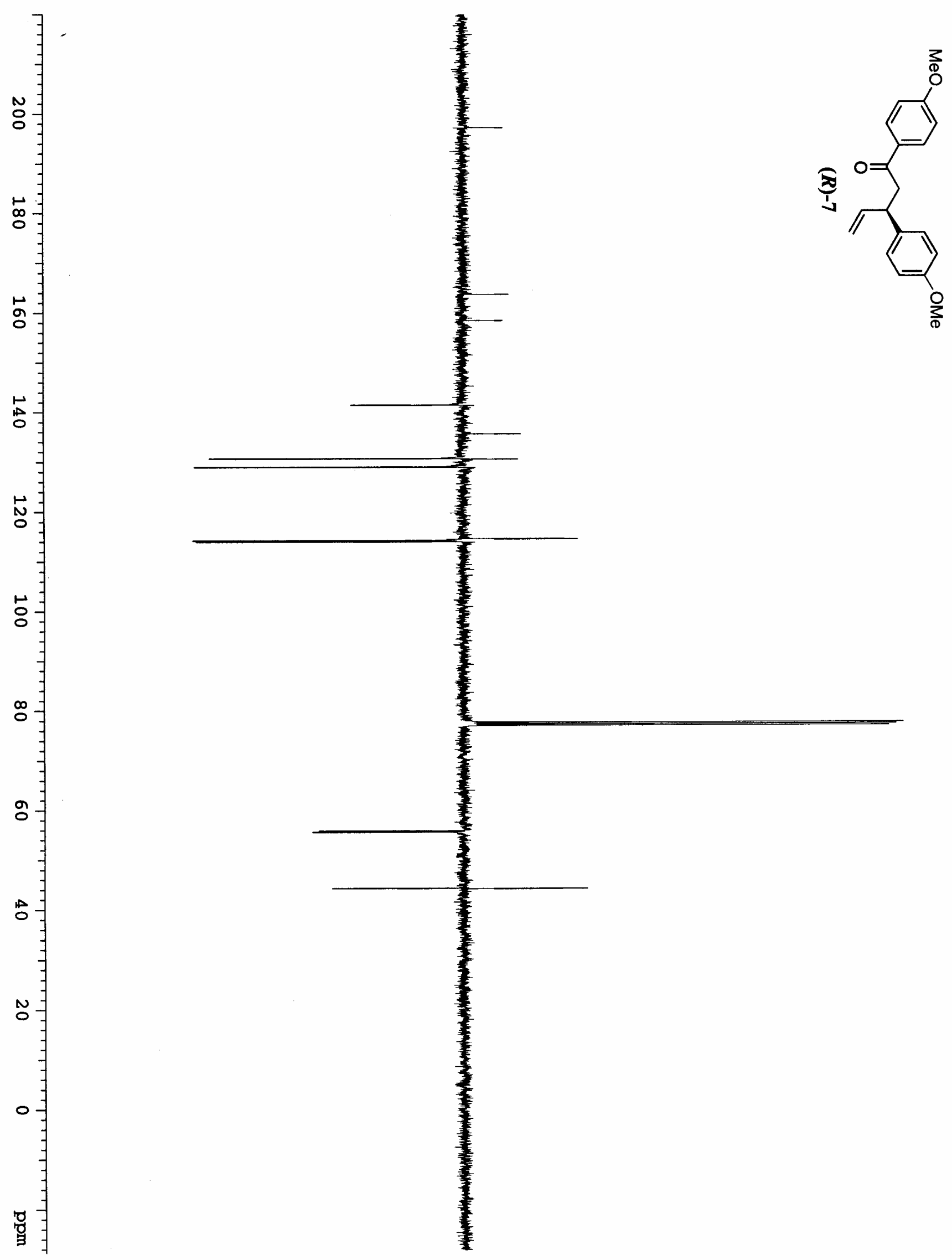


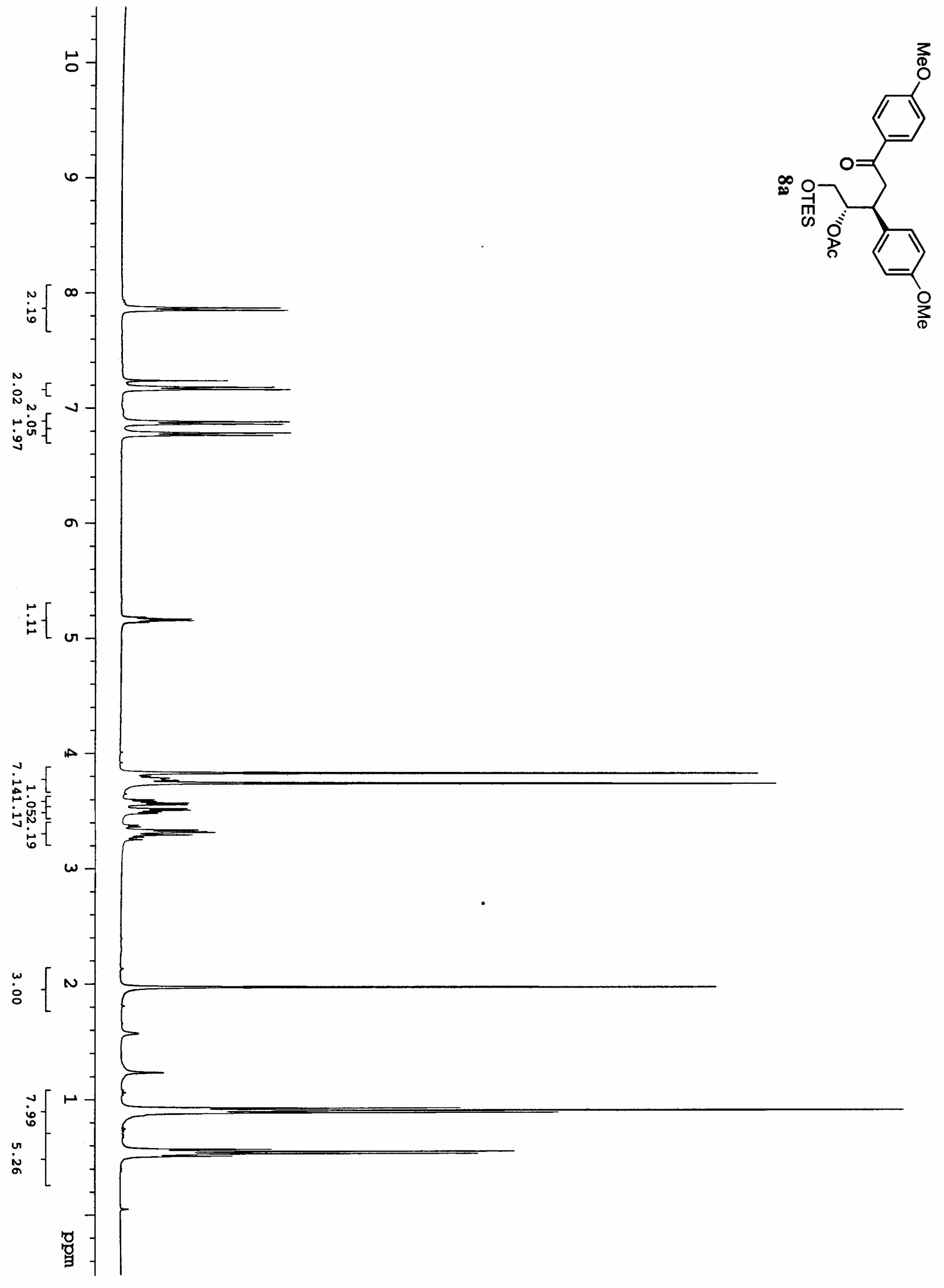



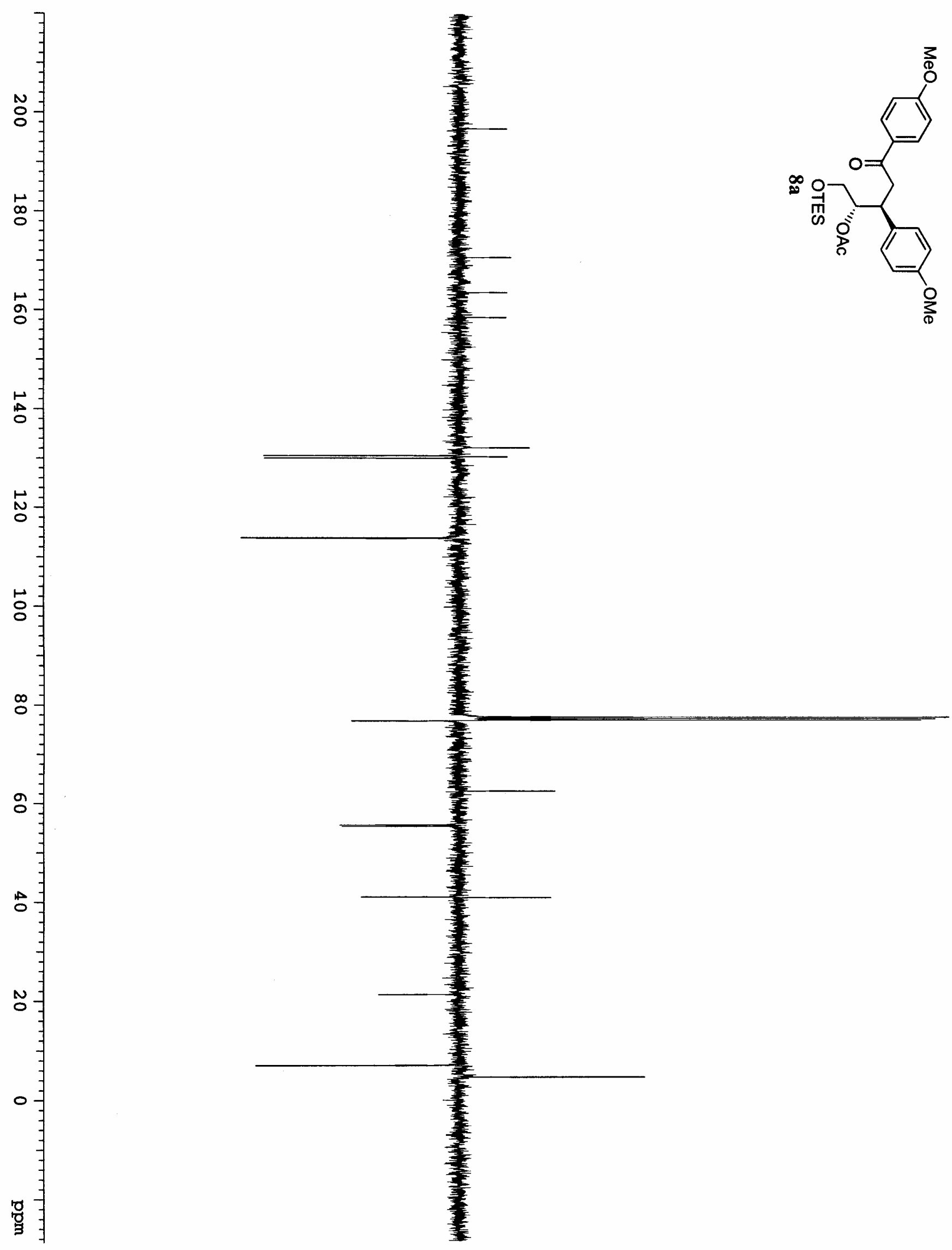


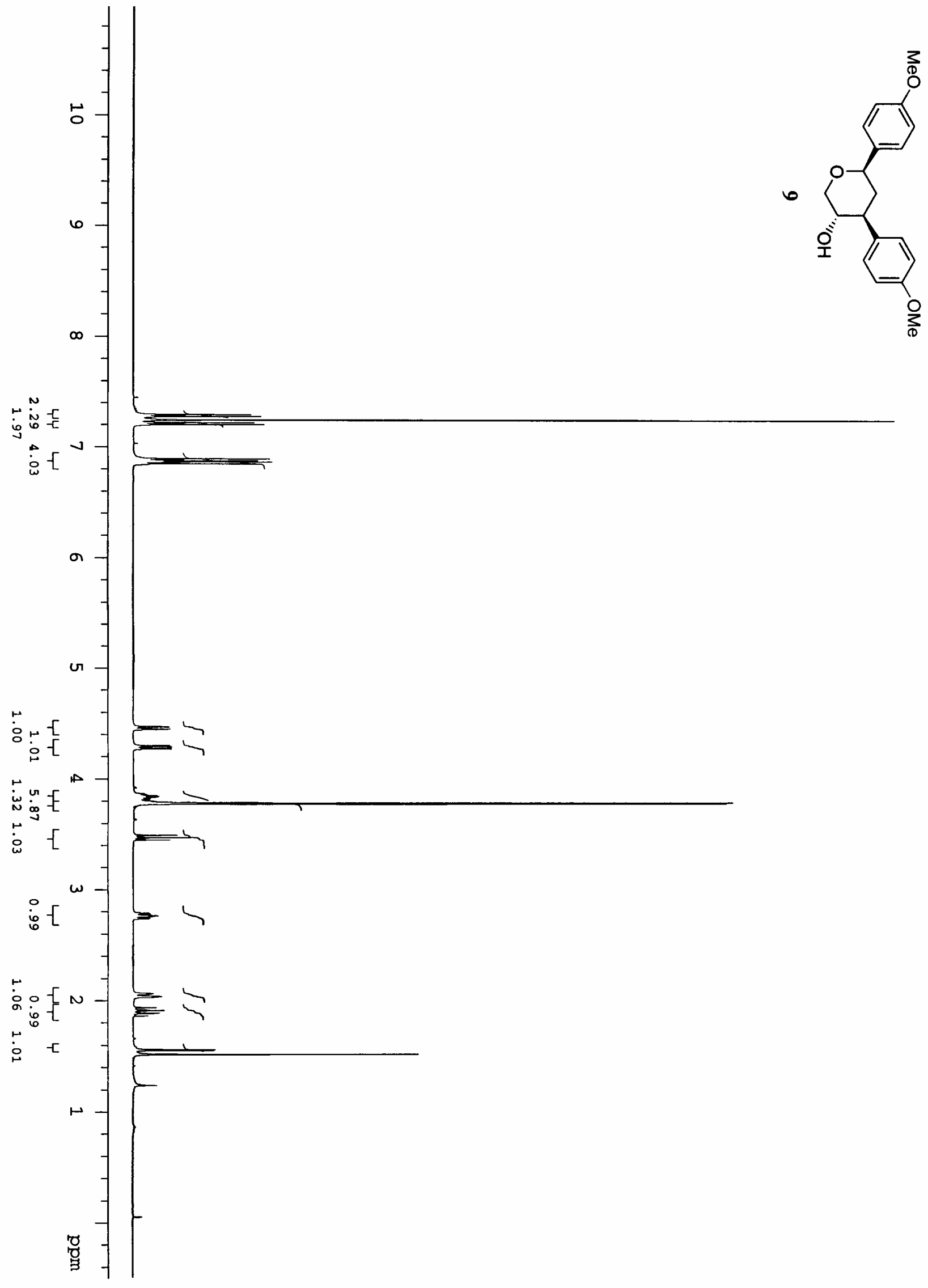



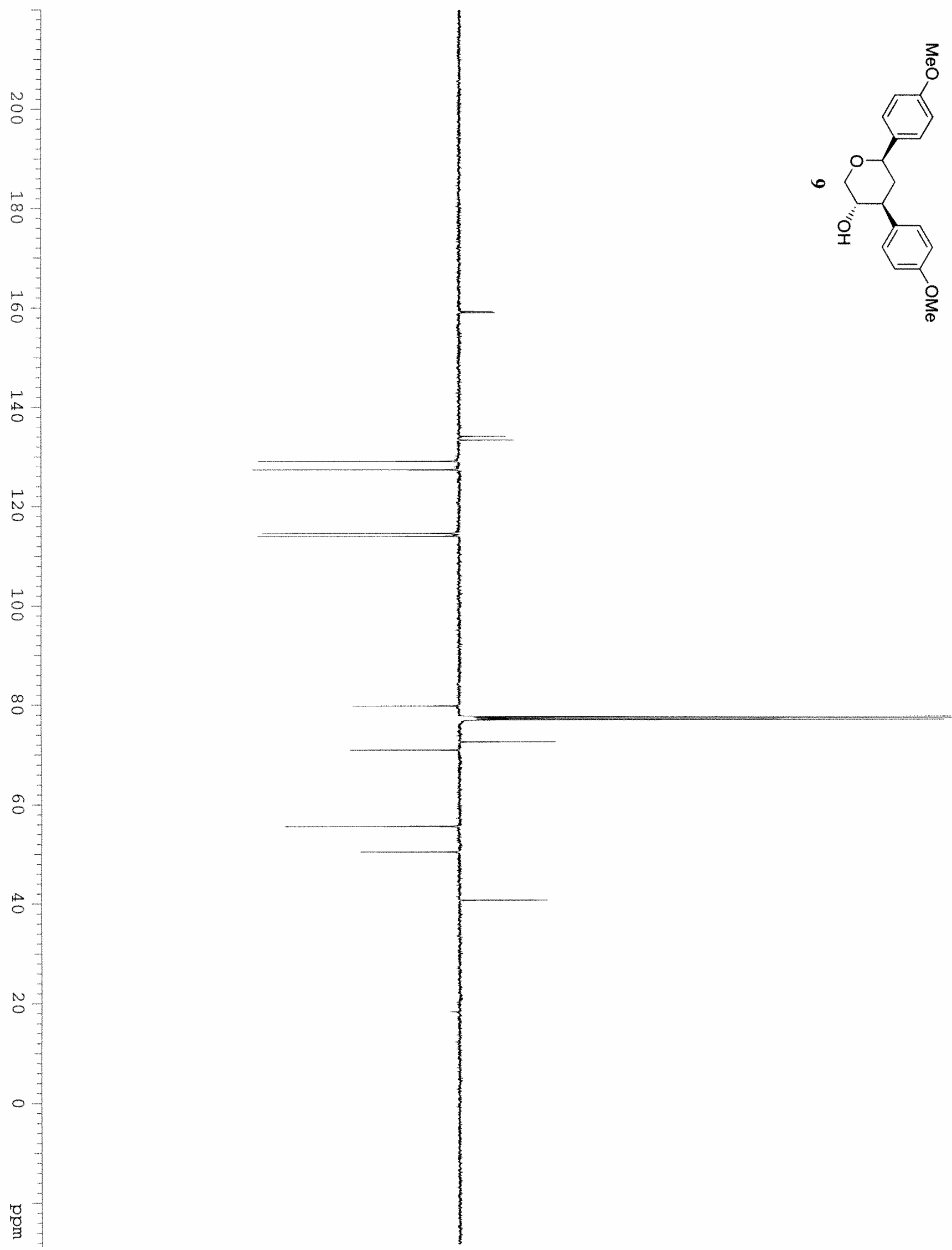


\section{Indiana University Molecular Structure Center}

Report 03087: $\quad \mathrm{C}_{21} \mathrm{H}_{19} \mathrm{NO}_{5}$

John C. Huffman

May 2, 2003
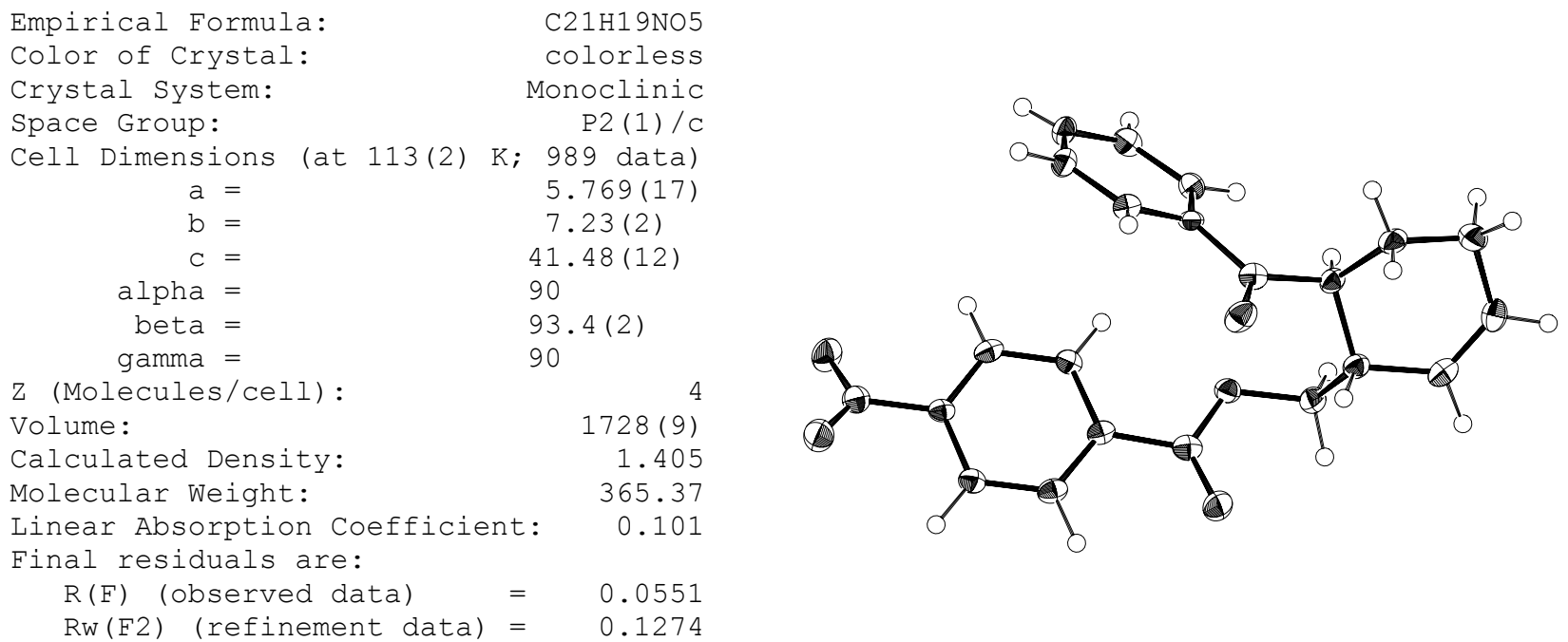

The sample was submitted by David K. Leahy, from the research group of Prof. P. A. Evans, Department of Chemistry, Indiana University. A typical colorless needle of approximate dimensions $0.70 \times 0.05 \times 0.05 \mathrm{~mm}$ onto the tip of a $0.1 \mathrm{~mm}$ diameter glass fiber and subsequently mounted on a SMART6000 (Bruker) and cooled to 113(2) K.

\section{Data collection}

A preliminary set of cell constants was calculated from reflections obtained from three nearly orthogonal sets of 20 frames. The data collection was carried out using graphite monochromated Mo K $\alpha$ radiation with a frame time of 15 seconds and a detector distance of $5.0 \mathrm{~cm}$. A randomly oriented region of a sphere in reciprocal space was surveyed. Two sections of 606 frames were collected with $0.30^{\circ}$ steps in $\omega$ at different $\phi$ settings with the detector set at $-43^{\circ}$ in $2 \theta$. Final cell constants were calculated from the xyz centroids of 989 strong reflections from the actual data collection after integration (SAINT).

\section{Structure solution and refinement}

Intensity statistics and systematic absences suggested the centrosymmetric space group $\mathrm{P} 2{ }_{1} / \mathrm{c}$ and subsequent solution and refinement confirmed this choice. The structure was solved using SHELXS-97 and refined with SHELXL-97. A direct-methods solution was calculated which provided most non-hydrogen atoms from the E-map. Full-matrix least squares / difference Fourier cycles were performed which located the remaining non-hydrogen atoms. All nonhydrogen atoms were refined with anisotropic displacement parameters. All hydrogen atoms were located in subsequent Fourier maps and included as isotropic contributors in the final cycles of refinement.

Complete data are available at http://bl-chem-iumsc110.chem.indiana.edu/recipnet/lab/sample.jsp?sampleId=59057817 